\title{
ON THE SOLUTIONS OF QUASI-LINEAR ELLIPTIC PARTIAL DIFFERENTIAL EQUATIONS*
}

BY

CHARLES B. MORREY, JR.

In this paper, we are concerned with the existence and differentiability properties of the solutions of "quasi-linear" elliptic partial differential equations in two variables, i.e., equations of the form

$$
\begin{aligned}
& A(x, y, z, p, q) r+2 B(x, y, z, p, q) s+C(x, y, z, p, q) t=D(x, y, z, p, q) \\
& A C-B^{2}>0, A>0\left(p=\frac{\partial z}{\partial x}, q=\frac{\partial z}{\partial y}, \quad r=\frac{\partial^{2} z}{\partial x^{2}}, s=\frac{\partial^{2} z}{\partial x \partial y}, t=\frac{\partial^{2} z}{\partial y^{2}}\right) .
\end{aligned}
$$

These equations are special cases of the general elliptic equation

$$
\phi(x, y, z, p, q, r, s, t)=0, \phi_{r} \phi_{t}-\phi_{s}^{2}>0, \phi_{r}>0 .
$$

The literature concerning these equations being very extensive, we shall not attempt to give a complete list of references. The starting point for many more modern researches has been the work of S. Bernstein, $\dagger$ who was the first to prove the analyticity of the solutions of the general equation with $\phi$ analytic and who was able to obtain a priori bounds for the second and higher derivatives of $z$ in the quasi-linear type in terms of the bounds of $|z|,|p|,|q|$ and the derivatives of the coefficients. He was also able to prove the existence of the solution of the quasi-linear equation in some very general cases. He assumed that all the data were analytic. However, his papers are very complicated and certain details require modification. On account of the results of J. Horn, L. Lichtenstein, and many others, $\ddagger$ the restriction of analyticity has been removed. Some very interesting modern work has been done by Leray and Schauder§ in a paper in which they develop a general theory of nonlinear functional equations and apply their results to quasi-linear equations.

* Presented to the Society, April 11, 1936; received by the editors February 9, 1937.

$\dagger$ Particularly the papers: Sur la nature analytique des solutions des équations aux dérivées partielles $d u$ second ordre, Mathematische Annalen, vol. 59 (1904), pp. 20-76 and Sur la généralization $d u$ problème de Dirichlet, Mathematische Annalen, vol. 69 (1910), pp. 82-136.

¥ For an account of some of these results, see the article by L. Lichtenstein on the theory of elliptic partial differential equations in the Encyklopädie der Mathematischen Wissenschaften, vol. II $3^{2}$, pp. $1280-1334$.

$\S$ Jean Leray and Jules Schauder, Topologie et équations fonctionelles, Annales Scientifiques de l'École Normale Supérieure, vol. 51 (1934), pp. 45-78. 
Schauder* has also obtained good a priori bounds for the solutions (and their derivatives) of linear elliptic equations in any number of variables.

In the present paper, an elliptic pair of linear partial differential equations of the form

(1) $v_{x}=-\left(b_{2} u_{x}+c u_{y}+e\right), v_{y}=a u_{x}+b_{1} u_{y}+d, 4 a c-\left(b_{1}+b_{2}\right)^{2} \geqq m>0$,

is studied. We assume merely that the coefficients are uniformly bounded and measurable. In such a general case, of course, the functions $u$ and $v$ do not possess continuous derivatives but are absolutely continuous in the sense of Tonelli with their derivatives summable with their squares (over interior closed sets). However, certain uniqueness, existence, and compactness theorems are demonstrated and the functions $u$ and $v$ are seen also to satisfy Hölder conditions. These results are immediately used to show that if $z(x, y)$ is a function which minimizes

$$
\iint_{R} f(x, y, z, p, q) d x d y, \quad\left(f_{p p} f_{q q}-f_{p q}^{2}>0, f_{p p}>0\right)
$$

among all functions (for which the integral may be defined) which take on the same boundary values as $z(x, y)$ and if $z(x, y)$ satisfies a Lipschitz condition on $R$, its first partial derivatives satisfy Hölder conditions. E. Hopf $\dagger$ has already shown that if $p$ and $q$ satisfy Hölder conditions, then the second derivatives satisfy Hölder conditions. In proving this fact, Hopf shows that if the coefficients in (1) satisfy Hölder conditions, the first partial derivatives of $u$ and $v$ satisfy Hölder conditions. The results of this paper concerning the system (1) together with Hopf's result yield very simple proofs of the existence of a solution of the quasi-linear equation in certain cases, a few of which are presented in $\S 6$.

The developments of this paper are entirely straightforward, being, for the most part, generalizations of known elementary results analogous to the step from Riemann to Lebesgue integration. The main tools by means of which the Hölder conditions of $u$ and $v$ in (1) are demonstrated are Theorems 1 and 2 of $\$ 2$ which state roughly that: if $\xi=\xi(x, y), \eta=\eta(x, y)$ is a $1-1$ differentiable transformation of a Jordan region $\bar{R}$ into another Jordan region $\bar{\Sigma}$ in which the ratio of the maximum to the minimum magnification is uniformly bounded, then the functions $\xi$ and $\eta$ and the functions $x(\xi, \eta)$ and $y(\xi, \eta)$ of the inverse satisfy Hölder conditions. Since these Hölder conditions

* J. Schauder, Über lineare elliptische Differentialgleichungen zweiter Ordnung, Mathematische Zeitschrift, vol. 38 (1933-34), pp. 257-282.

$\dagger$ E. Hopf, Zum analytischen Charakter der Lösungen regulärer zweidimensionaler Variationsprobleme, Mathematische Zeitschrift, vol. 30, pp. 404-413. 
are so important in the modern theory of elliptic equations, these two theorems may prove to be an important tool in this field.

We use the following notation: A function $\phi(x, y)$ is said to be of class $C^{(n)}$ if it is continuous together with its partial derivatives of the first $n$ orders. If $E$ is a point set, $\bar{E}$ denotes its closure and $E^{*}$ its boundary points. If $E$ and $F$ are point sets, the symbol $E \cdot F$ denotes their product, $E+F$ denotes their sum ( $E$ and $F$ need not be mutually exclusive), and $E \subset F$ means that $E$ is a subset of $F$. The symbol $C(P, r)$ denotes the open circular disc with center at $P$ and radius $r$.

1. Preliminary definitions and lemmas. Most of the definitions and lemmas of this section are either found in the literature or are easily deducible from known results. We include the material of this section for completeness.

Definition 1. A function $u(x, y)$ is said to be strictly absolutely continuous in the sense of Tonelli $\dagger$ (A.C.T.) on a closed rectangle $(a, c ; b, d)$ if it is continuous there and

(i) for almost all $X, \mathrm{a} \leqq X \leqq b, u(X, y)$ is absolutely continuous in $y$ on the interval $(c, d)$, and for almost all $Y, c \leqq Y \leqq d, u(x, Y)$ is absolutely continuous in $x$ on the interval $(a, b)$ and

(ii) $V_{c}^{(y) d}[u(X, y)]$ and $V_{a}^{(x) b}[u(x, Y)]$ are summable functions of $X$ and $Y$, respectively, $V_{c}^{(y) d}[u(X, y)]$, for instance, denoting the variation on $(c, d)$ of $u(X, y)$ considered as a function of $y$ alone. It is clear that these variations are lower semicontinuous in the large lettered variables.

Definition 2. A function $u(x, y)$ is said to be $A . C . T$. on a region $R$ (or $\bar{R}$ ) if it is continuous there and strictly A.C.T. on each closed interior rectangle.

Remark. Evans $\ddagger$ has shown that every continuous "potential function of its generalized derivatives" $\S$ is A.C.T. and conversely, so that his theorems concerning the former functions are applicable to the latter. Thus

LEMma $1 . \S$ If $u(x, y)$ is A.C.T. on $R$, then $\partial u / \partial x=u_{x}$ and $\partial u / \partial y=u_{y}$ exist almost everywhere in $R$ and are summable over every closed subregion of $R$.

LEMMA 2.ई If $x=x(s, t)$ and $y=y(s, t)$ is a 1-1 continuous transformation of class $C^{\prime}$ of a region $R$ of the $(x, y)$-plane into a region $\Sigma$ of the $(s, t)$-plane, and if $u(x, y)$ is A.C.T. on $R$, then the function $u[x(s, t), y(s, t)]$ is A.C.T. on $\Sigma$ and

$\dagger$ L. Tonelli, Sulla quadratura delle superficie, Atti della Reale Accademia dei Lincei, (6), vol. 3 (1926), pp. 357-362, 445-450, 633-638, 714-719.

$\ddagger$ G. C. Evans, Complements of potential theory, II, American Journal of Mathematics, vol. 55 (1933), pp. 29-49.

§ G. C. Evans, Fundamental points of potential theory, Rice Institute Pamphlets, vol. 7, No. 4 (1920), pp. 252-329. 


$$
u_{s}=u_{x} x_{s}+u_{y} y_{s}, \quad u_{t}=u_{x} x_{t}+u_{y} y_{t}
$$

almost everywhere.

Definition 3. Let $D$ be a region. By the expression almost all rectangles of $D$ we mean the totality of rectangles $a \leqq x \leqq b, c \leqq y \leqq d$ in $D$ for which $a$ and $b$ do not belong to some set of measure zero of values of $x$, and $c$ and $d$ do not belong to some set of measure zero of values of $y$. Naturally either or both sets of measure zero may be vacuous.

Definition 4. Let $\phi(D)$ be a function defined on almost all rectangles $D$ of a region $R$ such that whenever $D=D_{1}+D_{2}$, where $D_{1}$ and $D_{2}$ are admissible rectangles having only an edge in common, we have that $\phi(D)=\phi\left(D_{1}\right)$ $+\phi\left(D_{2}\right)$. We say that $\phi(D)$ is absolutely continuous on $R$ if for every $\epsilon>0$, there exists a $\delta>0$ such that for every sequence of non-overlapping admissible rectangles $\left\{D_{n}\right\}$ with $\sum\left[\right.$ meas $\left.\left(D_{n}\right)\right]<\delta$, we have $\sum\left|\phi\left(D_{n}\right)\right|<\epsilon$.

LeMma $3 . \dagger$ Let $f(x, y)$ be summable on $R$, let $D$ denote a rectangle $(a, c ; b, d)$ on which $f(x, y)$ is summable (this being almost all rectangles of $R$ ) and define

$$
\begin{aligned}
& \phi(D)=\int_{c}^{d}[f(b, y)-f(a, y)] d y=\int_{D^{*}} f d y, \\
& \psi(D)=\int_{a}^{b}[f(x, d)-f(x, c)] d x=-\int_{D^{*}} f d x .
\end{aligned}
$$

Then a necessary and sufficient condition that $f(x, y)$ be A.C.T. on $R$ is that $f(x, y)$ be continuous and $\phi(D)$ and $\psi(D)$ be absolutely continuous on each subregion $\Delta$ for which $\bar{\Delta} \subset R$. When this is true,

$$
\phi(D)=\iint_{D} \frac{\partial f}{\partial x} d x d y, \quad \psi(D)=\iint_{D} \frac{\partial f}{\partial y} d x d y
$$

for each rectangle in $R$.

Definition 5 . We say that a function $\phi(x, y)$ is of class $L_{p}$ on a region $R$ if $|\phi|^{p}$ is summable over $R$.

Definition 6. We say that a function $u(x, y)$ is of class $D_{\alpha}$ on $R$ or $\bar{R}$ if it is A.C.T. there and $\left|u_{x}\right|^{\alpha}$ and $\left|u_{y}\right|^{\alpha}$ are summable over every closed subregion of $R$.

LEMma 4. Let $\left\{\phi_{n}(x, y)\right\}, n=1,2, \cdots$, and $\phi(x, y)$ be of class $L_{p}$ on a rectangle $(a, c ; b, d)$ with

† A summable function $f(x, y)$ for which $\phi(D)$ and $\psi(D)$ are absolutely continuous on each subregion $\Delta$ of $R$ for which $\bar{\Delta} \subset R$ is said to be a "potential function of its generalized derivatives" and this lemma is essentially the theorem of Evans mentioned above (Complements of potential theory, loc. cit.). 


$$
\int_{a}^{b} \int_{c}^{d}\left|\phi_{n}(x, y)\right|^{p} d x d y \leqq G, \quad p>1,
$$

where $G$ is independent of $n$. Let

$$
\Phi_{n}(x, y)=\int_{a}^{x} \int_{c}^{\nu} \phi_{n}(\xi, \eta) d \xi d \eta, \quad \Phi(x, y)=\int_{a}^{x} \int_{c}^{\nu} \phi(\xi, \eta) d \xi d \eta
$$

and suppose that the sequence $\left\{\Phi_{n}(x, y)\right\}$ converges uniformly to $\Phi(x, y)$. Suppose also that $\left\{A_{n}(x, y)\right\}, n=1,2, \cdots$, and $A(x, y)$ are of class $L_{q}$ on $(a, c$; $b, d), q=p /(p-1)$, and that

Then

$$
\lim _{n \rightarrow \infty} \int_{a}^{b} \int_{c}^{d}\left|A_{n}-A\right|^{a} d x d y=0 .
$$

$$
\begin{aligned}
& \int_{a}^{b} \int_{c}^{d}|\phi|^{p} d x d y \leqq \underset{n \rightarrow \infty}{\liminf _{n}} \int_{a}^{b} \int_{c}^{d}\left|\phi_{n}\right|^{p} d x d y, \\
& \lim _{n \rightarrow \infty} \int_{a}^{b} \int_{c}^{d} A_{n} \phi_{n} d x d y=\int_{a}^{b} \int_{c}^{d} A \phi d x d y .
\end{aligned}
$$

Proof. The first conclusion is well known. $\dagger$

To prove (ii), choose $\epsilon>0$. For $n>N_{1}$, we see from the Hölder inequality that

$$
\begin{aligned}
\left|\int_{a}^{b} \int_{c}^{d}\left(A_{n}-A\right) \phi_{n} d x d y\right| \\
\qquad\left[\int_{a}^{b} \int_{c}^{d}\left|A_{n}-A\right|^{a} d x d y\right]^{1 / q}\left[\int_{a}^{b} \int_{c}^{d}\left|\phi_{n}\right|^{p} d x d y\right]^{1 / p}<\frac{\epsilon}{4} .
\end{aligned}
$$

Now, let $\left\{B_{k}(x, y)\right\}$ be a sequence of step functions $\ddagger$ such that

$$
\lim _{k \rightarrow \infty} \int_{a}^{b} \int_{c}^{d}\left|B_{k}-A\right|^{a} d x d y=0
$$

Then, for $k>K$ (independent of $n$ ),

$$
\left|\int_{a}^{b} \int_{c}^{d}\left(B_{k}-A\right) \phi_{n} d x d y\right|
$$

$\dagger$ For instance, this result may be obtained by the method of proof used in Theorem 7, 81 of the author's paper, $A$ class of representations of manifolds, I, American Journal of Mathematics, vol. 55 (1933), p. 693.

$\ddagger$ To form these, let $G_{k}$ denote the grating formed by all lines of the form $x=2^{-k} i, y=2^{-k} j$. We then define $B_{k}$ to be a properly chosen constant on the part of each square of $G_{k}$ which contains a point of the rectangle. 


$$
\leqq\left[\int_{a}^{b} \int_{c}^{d}\left|B_{k}-A\right| q d x d y\right]^{1 / q}\left[\int_{a}^{b} \int_{c}^{d}\left|\phi_{n}\right|^{p} d x d y\right]^{1 / p}<\frac{\epsilon}{4},
$$

$\left|\int_{a}^{b} \int_{c}^{d}\left(B_{k}-A\right) \phi d x d y\right|$

$$
\leqq\left[\int_{a}^{b} \int_{c}^{d}\left|B_{k}-A\right|^{a} d x d y\right]^{1 / q}\left[\int_{a}^{b} \int_{c}^{d}|\phi|^{p} d x d y\right]^{1 / p}<\frac{\epsilon}{4} .
$$

Now, let $k_{0}>K$. Then, for $n>N_{2}$,

$$
\left|\int_{a}^{b} \int_{c}^{d} B_{k_{0}}\left(\phi_{n}-\phi\right) d x d y\right| \leqq \sum_{i}\left|B_{k_{0}, i}\right| \cdot\left|\iint_{R_{i}}\left(\phi_{n}-\phi\right) d x d y\right|<\frac{\epsilon}{4},
$$

the $R_{i}$ being the rectangular subregions of $R$ over each of which $B_{k_{0}}$ is constant and $B_{k_{0}, i}$ being the value of $B_{k_{0}}$ on $R_{i}$.

Finally, if we let $N$ be the larger of $N_{1}$ and $N_{2}$, we see that

$$
\begin{aligned}
& \left|\int_{a}^{b} \int_{c}^{d}\left(A_{n} \phi_{n}-A \phi\right) d x d y\right| \\
& \leqq\left|\int_{a}^{b} \int_{c}^{d}\left(A_{n}-A\right) \phi_{n} d x d y\right|+\left|\int_{a}^{b} \int_{c}^{d}\left(A-B_{k_{0}}\right) \phi_{n} d x d y\right| \\
& \quad+\left|\int_{a}^{b} \int_{c}^{d} B_{k_{0}}\left(\phi_{n}-\phi\right) d x d y\right|+\left|\int_{a}^{b} \int_{c}^{d}\left(B_{k_{0}}-A\right) \phi d x d y\right|<\epsilon .
\end{aligned}
$$

This proves the lemma.

LEMMA 5. Let $\phi(x, y)$ be of class $L_{p}$ on $R, p \geqq 1$, and let $R_{h}$ be that subset of points $\left(x_{0}, y_{0}\right)$ of $R$ such that all points $(x, y)$ with $\left|x-x_{0}\right|<h,\left|y-y_{0}\right|<h$ are in $R$. Let

$$
\phi_{h}(x, y)=\frac{1}{4 h^{2}} \int_{x-h}^{x+h} \int_{y-h}^{y+h} \phi(\xi, \eta) d \xi d \eta, \quad h>0,
$$

be defined in $R_{h}$. Then $\phi_{h}(x, y)$ is continuous and of class $L_{p}$ on $R_{h}$ and

$$
\begin{aligned}
\iint_{R_{h}}\left|\phi_{h}\right|^{p} d x d y \leqq \iint_{R}|\phi|^{p} d x d y, \\
\lim _{h \rightarrow 0}\left[\iint_{R_{h}}\left|\phi_{h}\right|^{p} d x d y-\iint_{R}|\phi|^{p} d x d y\right] \\
=\lim _{h \rightarrow 0} \iint_{R_{h_{0}}}\left|\phi_{h}-\phi\right|^{p} d x d y=0, \quad h_{0}>0 .
\end{aligned}
$$


Proof. This lemma is well known. $\dagger$

Definition 7. Let $u(x, y)$ be defined on $R$ or $\bar{R}$. If $u$ is of class $D_{2}$ on $R$ or $\bar{R}$ with $\left|u_{x}\right|^{2}$ and $\left|u_{y}\right|^{2}$ summable over the whole of $R$, we define

$$
D(u)=\iint_{R}\left(u_{x}^{2}+u_{y}^{2}\right) d x d y .
$$

Otherwise we define $D(u)=+\infty$.

Lemma 6. Let $\left\{u_{n}(x, y)\right\}$ and $\left\{v_{n}(x, y)\right\}$ each be of class $D_{2}$ on $\bar{R}$ with $D\left(u_{n}\right)$ and $D\left(v_{n}\right) \leqq G$ independent of $n$, and suppose that $\left\{u_{n}(x, y)\right\}$ and $\left\{v_{n}(x, y)\right\}$ converge uniformly on $\bar{R}$ to functions $u(x, y)$ and $v(x, y)$, respectively. Let the sequences $\left\{a_{n}(x, y)\right\},\left\{b_{n}\right\},\left\{c_{n}\right\},\left\{d_{n}\right\},\left\{e_{n}\right\},\left\{f_{n}\right\},\left\{g_{n}\right\},\left\{h_{n}\right\},\left\{k_{n}\right\}$, and $\left\{l_{n}\right\}$ be measurable and uniformly bounded and suppose the sequences converge almost everywhere on $R$ to $a, b, c, d, e, f, g, h, k$, and $l$ respectively. Then

$$
\begin{aligned}
& u(x, y) \text { and } v(x, y) \text { are of class } D_{2} \text { on } \bar{R}, \\
& \begin{array}{r}
D(u) \leqq \\
\iint_{R}\left[\left(a u_{x}+b u_{y}+c v_{x}+d v_{y}+e\right)^{2}+\left(f u_{x}+g u_{y}+h v_{x}+k v_{y}+l\right)^{2}\right] d x d y \\
\quad \leqq \liminf _{n \rightarrow \infty} \iint_{R}\left[\left(a_{n} u_{n x}+b_{n} u_{n y}+c_{n} v_{n x}+d_{n} v_{n y}+e_{n}\right)^{2}\right. \\
\left.+\left(f_{n} u_{n x}+g_{n} u_{n y}+h_{n} v_{n x}+k_{n} v_{n y}+l_{n}\right)^{2}\right] d x d y .
\end{array}
\end{aligned}
$$

Proof. Conclusions (i) and (ii) are well known. $\dagger$

To prove (iii), let $M$ be the uniform bounds for $a_{n}$, etc., and let

$\phi_{n}=a_{n} u_{n x}+b_{n} u_{n y}+c_{n} v_{n x}+d_{n} v_{n y}+e_{n}, \quad \phi=a u_{x}+b u_{y}+c v_{x}+d v_{y}+e$,

$\psi_{n}=f_{n} u_{n x}+g_{n} u_{n y}+h_{n} v_{n x}+k_{n} v_{n y}+l_{n}, \quad \psi=f u_{x}+g u_{y}+h v_{x}+k v_{y}+l$.

Then, for each $h>0$, we see that $\phi_{n}{ }^{(h)}, \psi_{n}{ }^{(h)}, \phi^{(h)}$ and $\psi^{(h)}$ are uniformly bounded on $R_{h}$, the proof for $\phi_{n}{ }^{(h)}$, for instance, employing the Hölder inequality as follows:

$$
\begin{aligned}
\left|\phi_{n}^{(h)}\right| \leqq & \frac{1}{4 h^{2}}\left[\left|\int_{x-h}^{x+h} \int_{y-h}^{y+h} a_{n}(\xi, \eta) u_{n}(\xi, \eta) d \xi d \eta\right|\right. \\
& +\left|\int_{x-h}^{x+h} \int_{y-h}^{y+h} b_{n} u_{n \eta} d \xi d \eta\right|+\left|\int_{x-h}^{x+h} \int_{y-h}^{y+h} c_{n} v_{n} d \xi d \eta\right| \\
& \left.+\left|\int_{x-h}^{x+h} \int_{y-h}^{y+h} d_{n} v_{n \eta} d \xi d \eta\right|+\left|\int_{x-h}^{x+h} \int_{y-h}^{y+h} e_{n} d \xi d \eta\right|\right]
\end{aligned}
$$

† See Lemma 1, $\$ 1$ of the author's paper, loc. cit. 


$$
\begin{aligned}
& \leqq \frac{1}{4 h^{2}}\left\{\left[\int_{x-h}^{x+h} \int_{y-h}^{y+h}\left|a_{n}\right|^{2} d \xi d \eta\right]^{1 / 2}\left[\int_{x-h}^{x+h} \int_{y-h}^{y+h}\left|u_{n \xi}^{2}\right| d \xi d \eta\right]^{1 / 2}\right. \\
& \left.+*+*+*+4 M h^{2}\right\} \leqq \frac{2 M G^{1 / 2}}{h}+M,
\end{aligned}
$$

each $*$ denoting a term similar to the first term. By Lemma $4, \phi_{n h}$ and $\psi_{n h}$ converge at each point to $\phi$ and $\psi$ respectively. Hence,

$$
\begin{aligned}
\iint_{R_{h}}\left(\phi_{h}^{2}+\psi_{h}^{2}\right) d x d y & =\lim _{n \rightarrow \infty} \iint_{R_{h}}\left(\phi_{n h}^{2}+\psi_{n h}^{2}\right) d x d y \\
& \leqq \liminf _{n \rightarrow \infty} \iint_{R}\left(\phi_{n}^{2}+\psi_{n}^{2}\right) d x d y,
\end{aligned}
$$

using Lemma 5. The statement (iii) follows from Lemma 5 by letting $h \rightarrow 0$.

Lemma 7. Let $f$ and $\phi$ be of class $D_{2}$ on $R$, let $D$ be a Jordan subregion of $R$ such that $D^{*}$ is a rectifiable curve interior to $R$ on which $\phi$ is of bounded variation. Then $\partial(f, \phi) / \partial(x, y)$ is summable on $D$ and

$$
\int_{D^{*}} f d \phi=\iint_{D} \frac{\partial(f, \phi)}{\partial(x, y)} d x d y
$$

the line integral being the ordinary Stieltjes integral.

Proof. That the Jacobian is summable follows from the Schwarz inequality, since $f_{x}^{2}+f_{y}^{2}+\phi_{x}^{2}+\phi_{\nu}^{2}$ is summable over $D$.

Let $f_{h}$ and $\phi_{k}$ have their usual significance as average functions. If $|h|$, $|k|<\alpha, f_{h}$ and $\phi_{k}$ are defined on $D$ and moreover $f_{h}$ is of class $C^{\prime}$ if $h>0$ and $\phi_{k}$ is of class $C^{\prime}$ if $k>0$. Hence, by Green's theorem

$$
\int_{D^{*}} f_{h} d \phi_{k}=\iint_{D}\left(f_{h x} \phi_{k y}-f_{h y} \phi_{k x}\right) d x d y=-\int_{D^{*}} \phi_{k} d f_{h} .
$$

Letting $k$ tend to zero, and using Lemmas 4 and 5 and well known theorems on the Stieltjes integral, we see that

$$
\int_{D^{*}} f_{h} d \phi=-\int_{D^{*}} \phi d f_{h}=\iint_{D}\left(f_{h x} \phi_{\nu}-f_{h y} \phi_{x}\right) d x d y .
$$

The result follows by letting $h$ tend to zero.

2. Fundamental theorems on transformations. We state first

Definition 1 . We say that $u(x, y)$ satisfies a condition $A[\lambda ; M(a, d)]$ on $R$ if it is of class $D_{2}$ on $R$ and 


$$
\begin{gathered}
\iint_{C(P, r)}\left(u_{x}^{2}+u_{y}^{2}\right) d x d y \leqq M(a, d)\left(\frac{r}{a}\right)^{\lambda}, \quad 0 \leqq r \leqq a, \\
P=(x, y) \varepsilon R, \quad \lambda>0,
\end{gathered}
$$

where $a>0, d>0$, and $a+d$ is the distance of $(x, y)$ from $R^{*}, M(a, d)$ depending on $a$ and $d$ and not on $(x, y)$.

Definition 2. We say that $u(x, y)$ satisfies a condition $B[\mu ; N(a, d)]$ on $R$ if

$$
\begin{gathered}
\left|u\left(x_{1}, y_{1}\right)-u\left(x_{2}, y_{2}\right)\right| \leqq N(a, d)\left(\frac{r}{a}\right)^{\mu}, \quad 0 \leqq r<a, \\
r=\left[\left(x_{2}-x_{1}\right)^{2}+\left(y_{2}-y_{1}\right)^{2}\right]^{1 / 2},
\end{gathered}
$$

provided that every point on the segment joining $\left(x_{1}, y_{1}\right)$ to $\left(x_{2}, y_{2}\right)$ is at a distance $\geqq a / 2+d$ from $R^{*}$.

Lemma 1. Let $u(x, y)$ satisfy a condition $A[\lambda ; M(a, d)]$ on $R$. Then it also satisfies a condition $B[\lambda / 2 ; N(a, d)]$, where

$$
N(a, d)=8 \cdot 3^{-1 / 2} \cdot \lambda^{-1} \cdot[M(a / 2, d)]^{1 / 2} .
$$

Proof. First assume that $u(x, y)$ is of class $C^{\prime}$ on $R$. Let $P_{1}:\left(x_{1}, y_{1}\right)$ and $P_{2}:\left(x_{2}, y_{2}\right)$ be two points of $R$ which are such that every point on the segment joining them is at a distance $\geqq a / 2+d$ from $R^{*}$. Next, choose axes so that $P_{1}$ is the origin, and $P_{2}$ is the point $\left(2^{-1 / 2} \cdot r, 2^{-1 / 2} \cdot r\right)$. Then each square of the form

$$
\begin{aligned}
0 \leqq x \leqq 2^{-1 / 2} \cdot r t, & 0 \leqq y \leqq 2^{-1 / 2} \cdot r t \quad \text { or } \\
2^{-1 / 2} \cdot r \cdot(1-t) & \leqq x \leqq 2^{-1 / 2} r, \quad 2^{-1 / 2} \cdot r \cdot(1-t) \leqq y \leqq 2^{-1 / 2} \cdot r
\end{aligned}
$$

is in a circle of radius $r t / 2$ whose center is at a distance $\geqq a / 2+d$ from $R^{*}$. Let $\alpha=2^{-1 / 2} r$; then

$$
\begin{array}{cc}
\int_{0}^{\alpha t} \int_{0}^{\alpha t}\left(u_{x}^{2}+u_{y}^{2}\right) d x d y \leqq M(a / 2, d)(r t / a)^{\lambda}, & 0 \leqq t \leqq 1, \\
\int_{\alpha-\alpha t}^{\alpha} \int_{\alpha-\alpha t}^{\alpha}\left(u_{x}^{2}+u_{y}^{2}\right) d x d y \leqq M(a / 2, d)(r t / a)^{\lambda}, & 0 \leqq t \leqq 1 .
\end{array}
$$

Now, for each $(x, y)$ with $0 \leqq x \leqq \alpha, 0 \leqq y \leqq \alpha$, we have

$$
\begin{aligned}
u(\alpha, \alpha)-u(0,0)= & x \int_{0}^{1} u_{x}(x t, y t) d t+y \int_{0}^{1} u_{y}(x t, y t) d t \\
& -(x-\alpha) \int_{0}^{1} u_{x}[\alpha+t(x-\alpha), \alpha+t(y-\alpha)] d t
\end{aligned}
$$




$$
-(y-\alpha) \int_{0}^{1} u_{y}[\alpha+t(x-\alpha), \alpha+t(y-\alpha)] d t .
$$

Integrating both sides of this equation with respect to $x$ and $y$, we obtain

$$
\begin{gathered}
u(\alpha, \alpha)-u(0,0)=\frac{1}{\alpha^{2}} \int_{0}^{\alpha} \int_{0}^{\alpha}\left\{\int_{0}^{1} x u_{x}(x t, y t) d t\right\} d x d y+* \\
-\frac{1}{\alpha^{2}} \int_{0}^{\alpha} \int_{0}^{\alpha}\left\{\int_{0}^{1}(x-\alpha) u_{x}[\alpha+t(x-\alpha), \alpha+t(y-\alpha)] d t\right\} d x d y-* \\
=\frac{1}{\alpha^{2}} \int_{0}^{1} \frac{1}{t^{3}}\left\{\int_{0}^{\alpha t} \int_{0}^{\alpha t} \xi u_{\xi}(\xi, \eta) d \xi d \eta+*\right. \\
\left.-\int_{\alpha-\alpha t}^{\alpha} \int_{\alpha-\alpha t}^{\alpha}(\xi-\alpha) u_{\xi}(\xi, \eta) d \xi d \eta-*\right\} d t,
\end{gathered}
$$

the last being obtained by suitable changes of variable; the $*$ denotes the term in $y$ or $\eta$ which is similar to the term preceding it. Using Schwarz's inequality on the interior terms, we obtain

$$
\begin{aligned}
|u(\alpha, \alpha)-u(0,0)| \leqq \int_{0}^{1} \alpha^{-2} t^{-3}\left\{\left[\int_{0}^{\alpha t} \int_{0}^{\alpha t} \xi^{2} d \xi d \eta\right]^{1 / 2}\left[\int_{0}^{\alpha t} \int_{0}^{\alpha t} u \xi^{2} d \xi d \eta\right]^{1 / 2}\right. \\
\left.\quad+*+\left[\int_{\alpha-\alpha t}^{\alpha} \int_{\alpha-\alpha t}^{\alpha}(\xi-\alpha)^{2} d \xi d \eta\right]^{1 / 2}\left[\int_{\alpha-\alpha t}^{\alpha} \int_{\alpha-\alpha t}^{\alpha} u_{\xi}^{2} d \xi d \eta\right]^{1 / 2}+*\right\} d t \\
\quad \leqq 4 \cdot 3^{-1 / 2}[M(a / 2, d)]^{1 / 2} \cdot(r / a)^{\lambda / 2} \int_{0}^{1} t^{\lambda / 2-1} d t \\
=8 \lambda^{-1} 3^{-1 / 2}[M(a / 2, d)]^{1 / 2} \cdot(r / a)^{\lambda / 2}
\end{aligned}
$$

If $u(x, y)$ is merely of class $D_{2}, u_{h}(x, y)$ is of class $C^{\prime}$ and we obtain the general result by letting $h$ tend to zero.

Definition 3. Let $T: \xi=\xi(x, y), \eta=\eta(x, y)$ be a 1-1 continuous transformation of a closed region $\bar{R}$ into a closed region $\bar{\Sigma}$, which is of class $C^{\prime}$ in $R$ with $\xi_{x} \eta_{y}-\xi_{y} \eta_{x} \neq 0$. Let $\left(x_{0}, y_{0}\right)$ be a point of $R, x=x(\sigma), y=y(\sigma)(\sigma$ arc length) be a regular curve such that $x\left(\sigma_{0}\right)=x_{0}, y\left(\sigma_{0}\right)=y_{0}, x^{\prime}\left(\sigma_{0}\right)=\cos \theta$, $y^{\prime}\left(\sigma_{0}\right)=\sin \theta$. If $\left(\xi_{0}, \eta_{0}\right)$ is the point of $\Sigma$ corresponding to $\left(x_{0}, y_{0}\right)$ and if $d s$ is the differential of arc length of the curve in $\Sigma$ corresponding to the above, we define the magnification of $T$ at $\left(x_{0}, y_{0}\right)$ in the direction $\theta$ by $|d s / d \theta|$.

Remarks. Clearly this magnification depends only on $\left(x_{0}, y_{0}\right)$ and $\theta$ and not on the curve chosen. It is given by

$$
\left|\frac{d s}{d \sigma}\right|^{2}=E_{0} \cos ^{2} \theta+2 F_{0} \sin \theta \cos \theta+G_{0} \sin ^{2} \theta, \quad E_{0}=E\left(x_{0}, y_{0}\right), \text { etc. }
$$


The square of its maximum and minimum (with respect to $\theta$ ) at $P_{0}$ are given by

$$
\begin{aligned}
& \frac{1}{2}\left\{E_{0}+G_{0}+\left[\left(E_{0}+G_{0}\right)^{2}-4\left(E_{0} G_{n}-F_{0}{ }^{2}\right)\right]^{1 / 2}\right\}, \\
& \frac{1}{2}\left\{E_{0}+G_{0}-\left[\left(E_{0}+G_{0}\right)^{2}-4\left(E_{0} G_{0}-F_{0}^{2}\right)\right]^{1 / 2}\right\},
\end{aligned}
$$

respectively, so that the ratio of the maximum to the minimum magnification is

$$
\mu_{0}+\left(\mu_{0}^{2}-1\right)^{1 / 2}, \quad \mu_{0}=\frac{E_{0}+G_{0}}{2\left(E_{0} G_{0}-F_{0}^{2}\right)^{1 / 2}}
$$

at $P_{0}$. If this ratio is uniformly bounded in $R$, it is clear that the inverse transformation has the same property. In the foregoing remarks, $E, F$, and $G$ have their usual differential-geometric significance:

$$
E=\xi_{x}^{2}+\eta_{x}^{2}, \quad F=\xi_{x} \xi_{y}+\eta_{x} \eta_{y}, \quad G=\xi_{y}{ }^{2}+\eta_{y}{ }^{2} .
$$

TheOREM 1. Let $\bar{R}$ and $\bar{\Sigma}$ be two Jordan regions, $a, b$, and $c$ be three distinct points of $R^{*}$, and $\alpha, \beta$, and $\gamma$ be three distinct points of $\Sigma^{*}$. Let $\{T\}$ be a family of 1-1 continuous transformations of the form

$$
T: \xi=\xi(x, y), \quad \eta=\eta(x, y)
$$

which carry $\bar{R}$ into $\bar{\Sigma}$, which carry $a, b$, and $c$ into $\alpha, \beta$, and $\gamma$ respectively, and which satisfy the following hypotheses:

(1) each $T$ is of class $C^{\prime}$ within $R$ with $\xi_{x} \eta_{y}-\xi_{y} \eta_{x} \neq 0$, and

(2) the ratio of the maximum to the minimum magnification of each transformation at each point $(x, y)$ is $\leqq K$, which is independent of $x, y$, and $T$. Then there exist functions $M(a), N(a), P(a)$, and $m(a)$ which depend only on $K$, the regions $\bar{R}$ and $\bar{\Sigma}$, and the distribution of the points $a, b, c, \alpha, \beta$, and $\gamma$; and there exists a number $\lambda>0$ which depends only on $K$ such that

(i) $M(a), N(a), m(a)>0$ for $a>0, \lim _{a \rightarrow 0} M(a)=\lim _{a \rightarrow 0} N(a)=\lim _{a \rightarrow 0} P(a)$ $=\lim _{a \rightarrow 0} m(a)=0$,

(ii) all points of $R$ or $\Sigma$ which are at a distance $\geqq \rho>0$ from $R^{*}$ or $\Sigma^{*}$ correspond to points of the other region at a distance $\geqq m(\rho)$ from its boundary, and

(iii) the functions $\xi(x, y), \eta(x, y), x(\xi, \eta)$, and $y(\xi, \eta)$ all satisfy conditions of the form $A[2 \lambda ; M(a, d)]$ and $B[\lambda ; N(a, d)]$ with $M(a, d)=M(a), N(a, d)$ $=N(a)$, and the equicontinuity condition

$$
\begin{gathered}
\left|\phi\left(\alpha_{1}, \beta_{1}\right)-\phi\left(\alpha_{2}, \beta_{2}\right)\right| \leqq P(a), \quad\left[\left(\alpha_{2}-\alpha_{1}\right)^{2}+\left(\beta_{2}-\beta_{1}\right)^{2}\right]^{1 / 2}=a, \\
(\alpha, \beta)=(x, y) \text { or }(\xi, \eta), \phi=\xi, \eta, x, \text { or } y .
\end{gathered}
$$


Proof. Since the ratio of maximum to minimum magnification is $\leqq K$, it follows that

$$
\begin{aligned}
& \iint_{R}\left(\xi_{x}^{2}+\xi_{y}{ }^{2}+\eta_{x}{ }^{2}+\eta_{y}{ }^{2}\right) d x d y \leqq 2 K m(\Sigma), \\
& \iint_{\Sigma}\left(x_{\xi}{ }^{2}+x_{\eta}{ }^{2}+y_{\xi}{ }^{2}+y_{\eta}{ }^{2}\right) d \xi d \eta \leqq 2 K m(R) .
\end{aligned}
$$

From this, $\dagger$ follows the existence of the functions $P(a)$ and $m(a)$ satisfying the desired conditions.

Now, let $P_{0}$ belong to $R$, for example, being at a distance $a$ from $R^{*}$. The circle $\left(x-x_{0}\right)^{2}+\left(y-y_{0}\right)^{2} \leqq a^{2}$ is carried into a Jordan subregion of $\bar{\Sigma}$ which is surely a subset of the circle $\left(\xi-\xi_{0}\right)^{2}+\left(\eta-\eta_{0}\right)^{2} \leqq[P(a)]^{2},\left(\xi_{0}, \eta_{0}\right)$ being the correspondent of $\left(x_{0}, y_{0}\right)$. Let

Then

$$
\begin{gathered}
A(r)=\iint_{C\left(P_{0}, r\right)} J(x, y) d x d y=\int_{0}^{r} \int_{0}^{2 \pi} \rho J\left(x_{0}+\rho \cos \theta, y_{0}+\rho \sin \theta\right) d \rho d \theta, \\
0 \leqq r \leqq a, \quad J(x, y)=\left|\xi_{x} \eta_{y}-\xi_{y} \eta_{x}\right| .
\end{gathered}
$$

$$
\begin{aligned}
A^{\prime} & =\frac{d A}{d r}=r \int_{0}^{2 \pi} J\left(x_{0}+r \sin \theta, y_{0}+r \cos \theta\right) d \theta \geqq \frac{r}{K} \int_{0}^{2 \pi}\left(\xi_{s}^{2}+\eta_{s}^{2}\right) d \theta \\
& =\frac{1}{K} \int_{0}^{2 \pi r}\left(\xi_{s}^{2}+\eta_{s}^{2}\right) d s \geqq(2 K \pi r)^{-1}\left(\int_{0}^{2 \pi r}\left[\xi_{s}^{2}+\eta_{s}^{2}\right]^{1 / 2} d s\right)^{2} \\
& =(2 K \pi r)^{-1}\left[l\left(C_{r}\right)\right]^{2} \geqq \frac{2}{K r} \cdot A(r), \quad 0<r<a ;
\end{aligned}
$$

here, $s$ denotes arc length on the circle $\left(x-x_{0}\right)^{2}+\left(y-y_{0}\right)^{2}=r^{2}$, and $C_{r}$ is the curve in $\Sigma$ into which this circle is carried. Thus

and hence

$$
A^{\prime} / A \geqq 2 /(K r), \quad A(a) \leqq \pi[P(a)]^{2}
$$

Since

$$
A(r) \leqq \pi[P(a)]^{2}(r / a)^{2 / K}, \quad 0 \leqq r \leqq a .
$$

$$
J(x, y) \geqq(1 / 2 K) \cdot\left(\xi_{x}^{2}+\xi_{y}^{2}+\eta_{x}^{2}+\eta_{y}^{2}\right),
$$

we find that

$$
\iint_{C\left(P_{0}, r\right)}\left(\xi_{x}^{2}+\xi_{y}{ }^{2}+\eta_{x}{ }^{2}+\eta_{y}{ }^{2}\right) d x d y \leqq 2 K \pi[P(a)]^{2}(r / a)^{2 / K} .
$$

† See the author's paper, An analytic characterization of surfaces of finite Lebesgue area, I, American Journal of Mathematics, vol. 57 (1935), Theorem 1, §2, p. 699. 
Hence, we see that (iii) is satisfied (remembering Lemma 1) if we choose

$$
\lambda=1 / K, \quad M(a)=2 K \pi[P(a)]^{2}, \quad N(a)=8 \cdot 2^{1 / 2} \cdot 3^{-1 / 2} \pi^{1 / 2} K^{3 / 2} \cdot P(a / 2) .
$$

Definition 4. We say that a Jordan region $\bar{R}$ and three distinct points $a, b$, and $c$ of $R^{*}$ satisfy a condition $D\left(L, d_{0}\right)$ if (1) the distances $a b, a c$, and $b c$ are all $\geqq d_{0}>0$, (2) $R^{*}$ is rectifiable, and (3) if $P_{1}$ and $P_{2}$ are any two points of $R^{*}$, the ratio $\overparen{P_{1} P_{2}} \div \overline{P_{1} P_{2}} \leqq L$, where ${\widehat{P_{1} P_{2}}}_{2}$ is an arc of $R^{*}$ joining $P_{1}$ and $P_{2}$ which contains at most one of the points $a, b$, or $c$ in its interior.

THEOREM 2. Let the regions $\bar{R}$ and $\bar{\Sigma}$, the points $a, b, c, \alpha, \beta$, and $\gamma$, and the family $\{T\}$ of transformations satisfy the hypotheses of Theorem 1 and suppose $(\bar{R} ; a, b, c)$ and $(\bar{\Sigma} ; \alpha, \beta, \gamma)$ satisfy a condition $D\left(L, d_{0}\right), d_{0}>0$. Then the conclusions of Theorem 1 hold and, in addition, there exists a number $M$ depending only on $K, L, d_{0}$ and the areas $m(\Sigma)$ and $m(R)$, and a number $\mu>0$ depending only on $K$ and $L$ such that

$$
\begin{aligned}
& \iint_{C\left(P_{0}, r\right) \cdot R}\left(\xi_{x}^{2}+\xi_{y}{ }^{2}+\eta_{x}{ }^{2}+\eta_{y}{ }^{2}\right) d x d y \leqq M r^{\mu}, \\
& \iint_{C\left(P_{0}, r\right) \cdot \Sigma}\left(x_{\xi}{ }^{2}+x_{\eta}{ }^{2}+y_{\xi}{ }^{2}+y_{\eta}{ }^{2}\right) d \xi d \eta \leqq M r^{\mu},
\end{aligned}
$$

for any point $P_{0}$ in the plane.

Proof. We need to prove only the last statement. Let $P_{0}$ be a point in the plane and let $0<r \leqq d_{0} / 2$. Then the set $C\left(P_{0}, r\right) \cdot R$ is vacuous or consists of a finite or denumerable number of Jordan regions $r_{n}$, the boundary of each of which consists of (1) a finite or denumerable set of arcs of $C^{*}\left(P_{0}, r\right) \cdot \bar{R}$, (2) a finite or denumerable number of arcs of $R^{*}$, and (3) points of $R^{*}$ which are limit points of all of these arcs. All the points of (2) and (3) are on one of the arcs $\overparen{a b c}, \overparen{b c a}$, or $\overparen{c a b}$ of $R^{*}$, say $\overparen{a b c}$. Clearly $r_{n}^{*}$ and $r_{n^{*}}{ }^{*}$ have at most one point in common if $n \neq n^{\prime}$. Let $E_{n, r}$ be the set (1) above for each $r_{n}$, let $E_{r}=\sum E_{r, n}$, let $\sigma_{n}$ be the region of $\Sigma$ corresponding to $r_{n}$, let $\sigma=\sum \sigma_{n}$, let $C_{r, n}=\sigma_{n}^{*}$, let $C_{r}=\sum C_{r, n}$, let $\Gamma_{r, n}$ be the totality of arcs corresponding to $E_{r, n}$, and let $\Gamma_{r}=\sum \Gamma_{r, n}$. Clearly $C_{r, n} \cdot C_{r, n^{\prime}}$ is at most one point if $n \neq n^{\prime}$. Let $l\left(C_{r}\right)=\sum l\left(C_{r, n}\right)$, let $l\left(\Gamma_{r, n}\right)$ be the sum of the lengths of all the arcs of $\Gamma_{r, n}$ and let $l\left(\Gamma_{r}\right)=\sum l\left(\Gamma_{r, n}\right)$. It is clear that

$$
\left[l\left(C_{r}\right)\right]^{2} \geqq 4 \pi m(\sigma) .
$$

Consider an $r_{n}$, and the closed set $R^{*} \cdot r_{n}^{*}$. Proceeding along the arc $(\widehat{a b c})$, there is a first point $P_{n}$ and a last point $Q_{n}$ of this set. Then, there is an arc of $E_{r, n}$ joining $P_{n}$ to $Q_{n}$. Hence if $\Pi_{n}$ and $K_{n}$ are the corresponding points 
of $\Sigma^{*}$, they are on the arc $\widehat{\alpha \beta \gamma}$ and there is an $\operatorname{arc}$ of $\Gamma_{r, n}$ joining them. Now $l\left(\Pi_{n}, K_{n}\right)$ (arc of $\left.\alpha \widehat{\beta \gamma}\right) \leqq L$ times the length of this arc of $\Gamma_{r, n}$. Hence it is easy to see that

$$
l\left(\Gamma_{r, n}\right) \geqq \frac{1}{L+1} l\left(C_{r, n}\right), \quad l\left(\Gamma_{r}\right) \geqq \frac{1}{L+1} l\left(C_{r}\right) .
$$

Any of the above sets may be null and for a set of measure zero of values of $r$, $l\left(C_{r}\right)$ and $l\left(\Gamma_{r}\right)$ may be infinite.

We may now proceed as in Theorem 1 . Let

$$
\begin{aligned}
A(r) & =\iint_{C\left(P_{0}, r\right) \cdot R} J(x, y) d x d y=\int_{0}^{r}\left[\rho \int_{E_{\rho}} J\left(x_{0}+\rho \cos \theta, y_{0}+\rho \sin \theta\right) d \theta\right] d \rho, \\
0 & \leqq r \leqq d_{0} / 2, \quad J(x, y)=\left|\xi_{x} \eta_{y}-\xi_{y} \eta_{x}\right|,
\end{aligned}
$$

(the integral being zero if the field of integration is null). Then

$$
\begin{aligned}
A^{\prime}(r) & =r \int_{E_{r}} J\left(x_{0}+r \cos \theta, y_{0}+r \sin \theta\right) d \theta \geqq \frac{1}{K} \int_{E_{r}}\left(\xi_{\theta}^{2}+\eta_{s}^{2}\right) d s \\
& \geqq(2 K \pi r)^{-1}\left[\int_{E_{r}}\left(\xi_{s}^{2}+\eta_{s}^{2}\right)^{1 / 2} d s\right]^{2}=(2 K \pi r)^{-1}\left[l\left(\Gamma_{r}\right)\right]^{2} \\
& \geqq(2 K \pi r)^{-1}(L+1)^{-2}\left[l\left(C_{r}\right)\right]^{2} \geqq 2 K^{-1}(L+1)^{-2} r^{-1} \cdot A(r) .
\end{aligned}
$$

Also $A\left(d_{0} / 2\right) \leqq m(\Sigma)$. Hence as before

$$
\begin{gathered}
A(r) \leqq m(\Sigma) \cdot\left(2 r / d_{0}\right)^{2 / K(L+1)^{2}} \\
\iint_{C\left(P_{0}, r\right)}\left(\xi_{x}{ }^{2}+\xi_{y}{ }^{2}+\eta_{x}{ }^{2}+\eta_{y}{ }^{2}\right) d x d y \leqq 2 K m(\Sigma)\left(2 r / d_{0}\right)^{2 / K(L+1)^{2}} .
\end{gathered}
$$

Since $A(r) \leqq m(\Sigma)$ for all values of $r$, the theorem follows.

LEMMA 2. Let $a, b_{1}, b_{2}, c, d$, and e be measurable functions defined on a bounded region $R$ with $|a|,\left|b_{1}\right|,\left|b_{2}\right|,|c|,|d|,|e| \leqq M \geqq 1$ on $R$. Then there exist sequences $\left\{a_{n}\right\},\left\{b_{1, n}\right\},\left\{b_{2, n}\right\},\left\{c_{n}\right\},\left\{d_{n}\right\}$, and $\left\{e_{n}\right\}$ which are analytic on $\bar{R}$ and uniformly bounded and which converge almost everywhere on $R$ to $a, b_{1}, b_{2}, c, d$, and e respectively. If $b_{1}=b_{2}$, we may choose $b_{1, n}=b_{2, n}$ for each $n$. If $b_{1}=b_{2}=b$ and $a c-b^{2} \geqq m>0$ on $R$, we may choose the sequences so that there exist numbers $\bar{M}$ and $\bar{m}>0$ such that

$$
\left|a_{n}\right|,\left|b_{n}\right|,\left|c_{n}\right|,\left|d_{n}\right|,\left|e_{n}\right| \leqq \bar{M}, \quad a_{n} c_{n}-b_{n}^{2} \geqq \bar{m}
$$

Further, if $a c-b^{2}=1$, the sequences may be chosen so that $a_{n} c_{n}-b_{n}{ }^{2}=1$.

Proof. Let $D$ be a region containing $\bar{R}$ in its interior and define $a=c=1$, 
$b_{1}=b_{2}=d=e=0$ in $D-R$. For $h$ sufficiently small, $a_{h}, b_{1 h}, b_{2 h}, c_{h}, d_{h}$, and $e_{h}$ are defined and continuous in a region containing $\bar{R}$ and all are numerically $\leqq M$.

Now suppose $b_{1}=b_{2}=b, a c-b^{2} \geqq m>0$. We know that $a c-b^{2}$ is the product of the maximum by the minimum (for $0 \leqq \theta \leqq 2 \pi,(x, y)$ fixed) of

$$
\begin{aligned}
f(x, y ; \theta) & =a \cos ^{2} \theta+2 b \sin \theta \cos \theta+c \sin ^{2} \theta \\
& =\frac{1}{2}[(a+c)+(a-c) \cos 2 \theta+2 b \sin 2 \theta] .
\end{aligned}
$$

Clearly $|f(x, y ; \theta)| \leqq 2 M$ so that the minimum above $\geqq m / 2 M$. Thus

$$
a_{h} c_{h}-b_{h}^{2} \geqq m^{2} / 4 M^{2}>0
$$

for each $h>0$ and all $(x, y)$ in $D_{h}$. The remainder of the proof is obvious.

LEMMA 3. Let $a, b$, and $c$ be analytic in a region $G$ which contains the Jordan region $\bar{R}$ in its interior and suppose $|a|,|b|,|c| \leqq M, a c-b^{2}=1, a>0$; let $\bar{\Sigma}$ be another Jordan region. Then there exists a unique 1-1 analytic map $\xi=(x, y), \eta=\eta(x, y)$ of $\bar{R}$ on $\Sigma$ which carries three given distinct points $p, q$, and $r$ on $R^{*}$ into three given distinct points $\pi, \kappa, \rho$ (arranged in the same order) on $\Sigma^{*}$, and which satisfies

$$
\eta_{x}=-\left(b \xi_{x}+c \xi_{y}\right), \quad \eta_{y}=a \xi_{x}+b \xi_{y} .
$$

The Jacobian does not vanish and the ratio of the maximum to the minimum magnification of the transformation is $\leqq M$ at each point.

Proof. Let $D$ be a Jordan region contained in $G$ and containing $\bar{R}$ whose boundary is a regular, analytic, simple, closed curve. It is known $\dagger$ that there exists a solution $X(x, y)$ of the equation

$$
\frac{\partial}{\partial x}\left(a X_{x}+b X_{y}\right)+\frac{\partial}{\partial y}\left(b X_{x}+c X_{y}\right)=0
$$

which takes on the values $X=x$ on $D^{*}$, which is analytic on $D$, and whose first derivatives do not vanish simultaneously. Clearly there exists an analytic conjugate function $Y(x, y)$ which satisfies the same equation and the relations

$$
Y_{x}=-\left(b X_{x}+c X_{y}\right), \quad Y_{y}=a X_{x}+b X_{y} .
$$

The equations $X=X(x, y), Y=Y(x, y)$ yield a 1-1 analytic map of ( $D$ and hence) $\bar{R}$ onto a region $\bar{\Delta}$ which carries $p, q$, and $r$ into three points $\pi^{\prime}, \kappa^{\prime}$, and $\rho^{\prime}$ arranged on $\Delta^{*}$ in the same order, and for which $X_{x} Y_{y}-X_{y} Y_{x} \neq 0$. If $\xi=\Xi(X, Y), \eta=\mathrm{H}(X, Y)$ is the conformal map of $\bar{\Delta}$ on $\bar{\Sigma}$ which carries $\pi^{\prime}, \kappa^{\prime}$, and $\rho^{\prime}$ into $\pi, \kappa$, and $\rho$ (respectively), it is easily seen that

† See Lichtenstein, loc. cit. 


$$
\xi=\xi(x, y)=\Xi[X(x, y), Y(x, y)], \eta=\eta(x, y)=\mathrm{H}[X(x, y), Y(x, y)]
$$

is a mapping of the desired type. That this mapping is unique follows from the fact that if $\xi=\xi^{\prime}(x, y), \eta=\eta^{\prime}(x, y)$ is another such map, then $\left(\xi^{\prime}, \eta^{\prime}\right)$ are related to $(\xi, \eta)$ by a conformal transformation with $\pi, \kappa$, and $\rho$ fixed; thus $\xi^{\prime} \equiv \xi, \eta^{\prime} \equiv \eta$.

LEMMA 4. Let $\xi=\xi(x, y), \eta=\eta(x, y)$ be a transformation defined on a region $R$ in which the functions $\xi$ and $\eta$ are of class $D_{2}$. Suppose also that

$$
\eta_{x}=-\xi_{y}, \quad \eta_{y}=\xi_{x}
$$

almost everywhere in $R$. Then the above map is conformal, i.e., $\xi$ and $\eta$ are conjugate harmonic functions.

Proof. Let $\bar{D}$ be a rectangle on the boundary of which $\eta(x, y)$ is absolutely continuous, such rectangles being almost all rectangles in $R$. Then, from Lemma 7, §1, it follows that

$$
\begin{aligned}
L\left(S_{D}\right) & =\iint_{D}\left(E G-F^{2}\right)^{1 / 2} d x d y=\frac{1}{2} \iint_{D}\left(\xi_{x}{ }^{2}+\xi_{y}{ }^{2}+\eta_{x}{ }^{2}+\eta_{y}{ }^{2}\right) d x d y \\
& =\iint_{D}\left(\xi_{x} \eta_{y}-\xi_{\nu} \eta_{x}\right) d x d y=\int_{D^{*}} \xi d \eta,
\end{aligned}
$$

$S_{D}$ being the surface $\xi=\xi(x, y), \eta=\eta(x, y),(x, y)_{\varepsilon} D, L\left(S_{D}\right)$ meaning its Lebesgue area. Since the Geöcze area $\dagger G(S)$ of any surface with this boundary curve must be at least as great as $L\left(S_{D}\right)$, and since $L(S) \geqq G(S)$ for every surface, we see that $S_{D}$ is a surface of minimum area bounded by its boundary curve. Hence $\xi(x, y)$ and $\eta(x, y)$ must be harmonic, since otherwise they could be replaced by the harmonic functions having the same boundary values to form a surface of smaller area bounded by the boundary of $S_{D}$.

TheOREM 3. Let $\bar{R}$ and $\bar{\Sigma}$ be Jordan regions, let $p, q$, and $r$ be distinct points on $R^{*}$ and let $\pi, \kappa$, and $\rho$ be distinct points arranged in the same order on $\Sigma^{*}$. Let $a, b$, and $c$ be bounded, measurable functions defined on $R$ :

$$
|a|,|b|,|c| \leqq M, \quad a c-b^{2}=1 .
$$

Then there exists a 1-1 continuous transformation $\xi=\xi(x, y), \eta=\eta(x, y)$ of $\bar{R}$ into $\bar{\Sigma}$

(i) which carries $p, q$, and $r$ into $\pi, \kappa$, and $\rho$ respectively,

(ii) which is such that $\xi(x, y), \eta(x, y), x(\xi, \eta)$, and $y(\xi, \eta)$ are of class $D_{2}$ on $\bar{R}$ and $\bar{\Sigma}, x=x(\xi, \eta), y=y(\xi, \eta)$ being the inverse transformation,

(iii) in which the conclusions of Theorem 1 apply to the functions $\xi(x, y)$,

† See the author's paper, loc. cit., pp. 696, 698. 
$\eta(x, y), x(\xi, \eta)$, and $y(\xi, \eta)$ and in which those of Theorem 2 also apply if $(\bar{R} ; p, q, r)$ and $(\bar{\Sigma} ; \pi, \kappa, \rho)$ satisfy a condition $D\left(L, d_{0}\right)$, and

(iv) in which the functions $\xi(x, y)$ and $\eta(x, y)$ satisfy

$$
\eta_{x}=-\left(b \xi_{x}+c \xi_{y}\right), \quad \eta_{y}=a \xi_{x}+b \xi_{y}
$$

almost everywhere on $R$.

Proof. Let $\left\{a_{n}\right\} \rightarrow a,\left\{b_{n}\right\} \rightarrow b,\left\{c_{n}\right\} \rightarrow c$ almost everywhere on $R$, the $a_{n}, b_{n}$, and $c_{n}$ being analytic on $\bar{R}$ and satisfying

$$
\left|a_{n}\right|,\left|b_{n}\right|,\left|c_{n}\right| \leqq \bar{M}, \quad a_{n} c_{n}-b_{n}^{2}=1 .
$$

Let $T_{n}: \xi=\xi_{n}(x, y), \eta=\eta_{n}(x, y)$ be the unique analytic transformations of $\bar{R}$ into $\bar{\Sigma}$ which carry $p, q$, and $r$ into $\pi, \kappa$, and $\rho$ respectively and which satisfy

$$
\eta_{n x}=-\left(b_{n} \xi_{n x}+c_{n} \xi_{n y}\right), \quad \eta_{n y}=a_{n} \xi_{n x}+b_{n} \xi_{n y} .
$$

Let $x=x_{n}(\xi, \eta), y=y_{n}(\xi, \eta)$ denote the inverses. These transformations satisfy the conditions of Theorem 1 and hence the conclusions of Theorem 1 and also of Theorem 2 if $(\bar{R} ; p, q, r)$ and $(\bar{\Sigma} ; \pi, \kappa, \rho)$ satisfy a condition $D\left(L, d_{0}\right)$; it is easily seen that the ratio of maximum to minimum magnification of $T_{n}$ is $\leqq \lambda_{n}+\left(\lambda_{n}{ }^{2}-1\right)^{1 / 2}, \lambda_{n}=\left(a_{n}+c_{n}\right) / 2$, and is therefore $\leqq 2 \bar{M}$. Hence a subsequence $\left\{n_{k}\right\}$ of the integers $\{n\}$ may be chosen so that $\left\{\xi_{n_{k}}\right\},\left\{\eta_{n_{k}}\right\},\left\{x_{n_{k}}\right\}$, and $\left\{y_{n_{k}}\right\}$ all converge uniformly on $\bar{R}$ and $\Sigma$ to functions $\xi(x, y), \eta(x, y)$, $x(\xi, \eta)$, and $y(\xi, \eta)$ respectively and $x=x(\xi, \eta), y=y(\xi, \eta)$ is the inverse of $T: \xi=\xi(x, y), \eta=\eta(x, y)$. Clearly $T$ is a $1-1$ continuous transformation of $\bar{R}$ into $\bar{\Sigma}$ in which $p, q$, and $r$ correspond to $\pi, \kappa$, and $\rho$ respectively.

Also, since the ratio of maximum to minimum magnification $\leqq 2 \bar{M}$, we have

$$
\begin{aligned}
& \iint_{R}\left(\xi_{n x}^{2}+\xi_{n y}^{2}+\eta_{n x}^{2}+\eta_{n y}^{2}\right) d x d y \leqq 4 \bar{M} m(\Sigma), \\
& \iint_{\Sigma}\left(x_{n \xi}^{2}+x_{n \eta}^{2}+y_{n \xi}^{2}+y_{n \eta}^{2}\right) d \xi d \eta \leqq 4 \bar{M} m(R),
\end{aligned}
$$

so that it follows from Lemma $6, \S 1$ that $\xi(x, y), \eta(x, y), x(\xi, \eta)$, and $y(\xi, \eta)$ are all of class $D_{2}$ on $\bar{R}$ and $\bar{\Sigma}$. Using the same lemma, we see that (iii) also holds and that

$$
\begin{aligned}
0 & \leqq \iint_{R}\left[\left(\eta_{x}+b \xi_{x}+c \xi_{y}\right)^{2}+\left(\eta_{y}-a \xi_{x}-b \xi_{y}\right)^{2}\right] d x d y \\
& \leqq \liminf _{n \rightarrow \infty} \iint_{R}\left[\left(\eta_{n x}+b_{n} \xi_{n x}+c_{n} \xi_{n y}\right)^{2}+\left(\eta_{n y}-a_{n} \xi_{n x}-b_{n} \xi_{n y}\right)^{2}\right] d x d y=0,
\end{aligned}
$$

so that (iv) is demonstrated. 
THEOREM 4. Let $(\bar{R} ; p, q, r),(\bar{\Sigma} ; \pi, \kappa, \rho), a, b$, and $c$ satisfy the hypotheses of Theorem 3 and let $T: \xi=\xi(x, y), \eta=\eta(x, y)$ be the transformation derived in that theorem. Then $T$ enjoys the following further properties:

(i) sets of measure zero and hence measurable sets of $R$ and $\Sigma$ correspond, and $\left(\xi_{x} \eta_{y}-\xi_{y} \eta_{x}\right)$ and $\left(x_{\xi} y_{\eta}-x_{\eta} y_{\xi}\right)$ are defined and $\neq 0$ except possibly on a set of measure zero in $R$ and $\Sigma$ respectively;

(ii) if $\phi(x, y)$ and $\psi(\xi, \eta)$ are summable on measurable subsets $D \subseteq R$ and $\Delta \subseteq \Sigma$, the functions $\phi[x(\xi, \eta), y(\xi, \eta)] \cdot\left(x_{\xi} y_{\eta}-x_{\eta} y_{\xi}\right)$ and $\psi[\xi(x, y), \eta(x, y)]$ $\cdot\left(\xi_{x} \eta_{y}-\xi_{y} \eta_{x}\right)$ are summable on $T(D)$ and $T^{-1}(\Delta)$ respectively, and

$$
\begin{array}{r}
\iint_{T(D)} \phi[x(\xi, \eta), y(\xi, \eta)] \cdot\left(x_{\xi} y_{\eta}-x_{\eta} y_{\xi}\right) d \xi d \eta=\iint_{D} \phi d x d y, \\
\iint_{T^{-1}(\Delta)} \psi \cdot\left(\xi_{x} \eta_{y}-\xi_{y} \eta_{x}\right) d x d y=\iint_{\Delta} \psi d \xi d \eta ;
\end{array}
$$

(iii) if $\phi(x, y)$ and $\psi(\xi, \eta)$ are of class $D_{2}$ on $R$ and $\Sigma$ respectively, then $\phi[x(\xi, \eta), y(\xi, \eta)]$ and $\psi[\xi(x, y), \eta(x, y)]$ are of class $D_{2}$ on $\Sigma$ and $R$ respectively and

$\phi_{\xi}=\phi_{x} x_{\xi}+\phi_{y} y_{\xi}, \quad \phi_{\eta}=\phi_{x} x_{\eta}+\phi_{y} y_{\eta}, \quad \phi_{x}=\phi_{\xi} \xi_{x}+\phi_{\eta} \eta_{x}, \quad \phi_{y}=\phi_{\xi} \xi_{y}+\phi_{\eta} \eta_{y}$, $\psi_{\xi}=\psi_{x} x_{\xi}+\psi_{y} y_{\xi}, \quad \psi_{\eta}=\psi_{x} x_{\eta}+\psi_{y} y_{\eta}, \quad \psi_{x}=\psi_{\xi} \xi_{x}+\psi_{\eta} \eta_{x}, \quad \psi_{y}=\psi_{\xi} \xi_{y}+\psi_{\eta} \eta_{y}$, almost everywhere;

(iv) $T$ is uniquely determined by (i), (ii), and (iv) of Theorem 3.

Proof. Let $O$ be an open set in $R$, let $O=\sum_{i=1}^{\infty} \bar{R}_{i}$ where the $\bar{R}_{i}$ are closed non-overlapping rectangles on each of which $\eta(x, y)$ is absolutely continuous, and let $S_{i}$ be the surface $S_{i}: \xi=\xi(x, y), \eta=\eta(x, y),(x, y)_{\varepsilon} \bar{R}_{i}$. Clearly $L\left(S_{i}\right)$ is merely the measure of the closed or open region ${ }_{i}$ or $\Sigma_{i}$ into which $\bar{R}_{i}$ or $R_{i}$ is carried, $\Sigma_{i}^{*}$ being a rectifiable curve. From Lemma $7, \S 1$, it follows that

$$
L\left(S_{i}\right)=m\left(\Sigma_{i}\right)=\int_{\Sigma_{i^{*}}} \xi d \eta=\int_{R_{i}^{*}} \xi d \eta=\iint_{R_{i}}\left(\xi_{x} \eta_{y}-\xi_{y} \eta_{x}\right) d x d y .
$$

Thus $\xi_{x} \eta_{y}-\xi_{y} \eta_{x} \geqq 0$, and if $\Omega=T(O)$, then

$$
m(\Omega)=\iint_{0}\left(\xi_{x} \eta_{y}-\xi_{y} \eta_{x}\right) d x d y .
$$

It follows very easily that a measurable set $E$ in $R$ is carried into one in $\Sigma$ and

$$
m[T(E)]=\iint_{E}\left(\xi_{x} \eta_{y}-\xi_{y} \eta_{x}\right) d x d y .
$$


The same proof establishes the fact that a measurable set $\Delta$ in $\Sigma$ is carried into a measurable set $D$ in $R$ and that

$$
m(D)=\iint_{\Delta}\left(x_{\xi} y_{\eta}-x_{\eta} y_{\xi}\right) d \xi d \eta .
$$

Hence the rest of (i) follows easily and we have proved (ii) for the case $\phi=\psi=1$.

Suppose $\phi$, for instance, is bounded. Let $\left\{\phi_{n}(x, y)\right\}$ be a sequence of step functions which are uniformly bounded and converge to $\phi$ almost everywhere. It is clear that the functions $\phi_{n}[x(\xi, \eta), y(\xi, \eta)] \cdot\left(x_{\xi} y_{\eta}-x_{\eta} y_{\xi}\right)$ and $\phi[x(\xi, \eta), y(\xi, \eta)] \cdot\left(x_{\xi} y_{\eta}-x_{\eta} y_{\xi}\right)$ are all summable and dominated by a summable function, and, for each $n$, it is clear from the above that

$$
\iint_{D} \phi_{n}(x, y) d x d y=\iint_{T(D)} \phi_{n}[x(\xi, \eta), y(\xi, \eta)]\left(x_{\xi} y_{\eta}-x_{\eta} y_{\xi}\right) d \xi d \eta .
$$

The result (ii) for $\phi$ follows by a passage to the limit, since $\phi_{n}[x(\xi, \eta), y(\xi, \eta)]$ converges almost everywhere on $\Sigma$ to $\phi[x(\xi, \eta), y(\xi, \eta)]$ and the latter function is certainly measurable. If $\phi$ is merely summable, let $\phi_{1}=\phi$ where $\phi \geqq 0$, $\phi_{1}=0$ elsewhere, $\phi_{2}=-\phi$ where $\phi<0$, and $\phi_{2}=0$ elsewhere, let $\phi_{1, N}=\phi_{1}$ if $\phi_{1} \leqq N, \phi_{1, N}=N$ elsewhere, $\phi_{2, N}=\phi_{2}$ if $\phi_{2} \leqq N, \phi_{2, N}=0$ elsewhere. Then it follows that the functions

$$
\phi_{1, N}\left(x_{\xi} y_{\eta}-x_{\eta} y_{\xi}\right), \quad \phi_{2, N}\left(x_{\xi} y_{\eta}-x_{\eta} y_{\xi}\right)
$$

form monotone non-decreasing sequences of non-negative summable functions converging almost everywhere to $\phi_{1} \cdot\left(x_{\xi} y_{\eta}-x_{\eta} y_{\xi}\right)$ and $\phi_{2} \cdot\left(x_{\xi} y_{\eta}-x_{\eta} y_{\xi}\right)$ respectively, their integrals remaining bounded. Hence (ii) follows.

To prove (iii), let $\phi(x, y)$ be of class $D_{2}$ on $R$, let $G$ be a subregion of $R$ for which $\bar{G} \subset R$, and let $\Gamma$ be the corresponding subregion of $\Sigma(\Gamma \subset \Sigma$ clearly). Now, let $\Delta$ be a rectangle of $\Gamma$ along which $x(\xi, \eta)$ and $y(\xi, \eta)$ are absolutely continuous. Then, using Lemma 7, $\$ 1$, we see that

$$
\begin{aligned}
\int_{\Delta^{*}} \phi[x(\xi, \eta), y(\xi, \eta)] d \eta & =\int_{D^{*}} \phi(x, y) d \eta=\iint_{D}\left(\phi_{x} \eta_{y}-\phi_{y} \eta_{x}\right) d x d y, \\
-\int_{\Delta^{\cdot}} \phi[x(\xi, \eta), y(\xi, \eta)] d \xi & =-\int_{D^{*}} \phi(x, y) d \xi=-\iint_{D}\left(\phi_{x} \xi_{y}-\phi_{y} \xi_{x}\right) d x d y .
\end{aligned}
$$

Clearly these relations follow for any rectangle $\Delta$ of $G$ so that $\phi$ is A.C.T. as a function of $\xi$ and $\eta$ by Lemma 3, $\$$. Using the same lemma, it follows that

$$
\iint_{\Omega} \frac{\partial \phi}{\partial \xi} d \xi d \eta=\iint_{o} \frac{\partial \phi}{\partial \xi} \cdot\left(\xi_{x} \eta_{y}-\xi_{\nu} \eta_{x}\right) d x d y=\iint_{o}\left(\phi_{x} \eta_{\nu}-\phi_{y} \eta_{x}\right) d x d y,
$$




$$
\iint_{\Omega} \frac{\partial \phi}{\partial \eta} d \xi d \eta=\iint_{0} \frac{\partial \phi}{\partial \eta} \cdot\left(\xi_{x} \eta_{y}-\xi_{y} \eta_{x}\right) d x d y=\iint_{0}\left(-\phi_{x} \xi_{y}+\phi_{y} \xi_{x}\right) d x d y,
$$

for every open set $\Omega$ in $\Sigma$ with $\bar{\Omega} \subset \Sigma$. Hence, almost everywhere in $R$ and $\Sigma$,

$$
\begin{aligned}
\phi_{x} \eta_{y}-\phi_{y} \eta_{x} & =\phi_{\xi} \cdot\left(\xi_{x} \eta_{y}-\xi_{y} \eta_{x}\right), \\
-\phi_{x} \xi_{y}+\phi_{y} \xi_{x} & =\phi_{\eta} \cdot\left(\xi_{x} \eta_{y}-\xi_{y} \eta_{x}\right) .
\end{aligned}
$$

Since $\xi_{x} \eta_{y}-\xi_{y} \eta_{x} \neq 0$ except on a set of measure zero, it follows that

$$
\phi_{x}=\phi_{\xi} \xi_{x}+\phi_{\eta} \eta_{x}, \quad \phi_{y}=\phi_{\xi} \xi_{y}+\phi_{\eta} \eta_{y},
$$

almost everywhere on $R$ and $\Sigma$. Setting $\phi=x$ and $y$ in turn, we obtain the relations

$$
x_{\xi} \xi_{x}+x_{\eta} \eta_{x}=1, \quad x_{\xi} \xi_{y}+x_{\eta} \eta_{y}=0, \quad y_{\xi} \xi_{x}+y_{\eta} \eta_{x}=0, \quad y_{\xi} \xi_{y}+y_{\eta} \eta_{y}=1
$$

almost everywhere, from which it follows that

$$
\phi_{\xi}=\phi_{x} x_{\xi}+\phi_{y} y_{\xi}, \quad \phi_{\eta}=\phi_{x} x_{\eta}+\phi_{y} y_{\eta}
$$

almost everywhere on $R$ and $\Sigma$; the relations for $\psi$ are proved similarly. It follows that

$$
\left(\xi_{x} \eta_{y}-\xi_{\nu} \eta_{x}\right) \cdot\left(x_{\xi} y_{\eta}-x_{\eta} y_{\xi}\right)=1
$$

almost everywhere on $R$ and $\Sigma$.

To show that $\phi$ is of class $D_{2}$ in $(\xi, \eta)$ we see that

$$
\left(\phi_{\xi}^{2}+\phi_{\eta}^{2}\right) \cdot\left(\xi_{x} \eta_{y}-\xi_{y} \eta_{x}\right)=a \phi_{x}^{2}+2 b \phi_{x} \phi_{y}+c \phi_{y}{ }^{2} \leqq 2 \bar{M}\left(\phi_{x}{ }^{2}+\phi_{y}{ }^{2}\right),
$$

using the relations above and the relations $\eta_{x}=-\left(b \xi_{x}+c \xi_{y}\right), \eta_{y}=a \xi_{x}+b \xi_{y}$, $a c-b^{2}=1$. The fact for $\psi$ as a function of $(x, y)$ can be obtained from the above and the fact that

$$
a \psi_{x}^{2}+2 b \psi_{x} \psi_{y}+a \psi_{y}^{2} \geqq \frac{1}{2 \bar{M}}\left(\psi_{x}^{2}+\psi_{y}^{2}\right) .
$$

To show (iv), let $\xi=\xi^{\prime}(x, y), \eta=\eta^{\prime}(x, y)$ be another map of $\bar{R}$ into $\bar{\Sigma}$ satisfying (i), (ii), and (iv) of Theorem 3. Then $\xi^{\prime}$ and $\eta^{\prime}$ are of class $D_{2}$ in $\xi$ and $\eta$ and all the rules for differentiation apply. We see that $\eta_{\eta}^{\prime}=\xi_{\xi}^{\prime}$ and $\eta_{\xi}^{\prime}=-\xi_{\eta}^{\prime}$ almost everywhere on $\Sigma$ so the map $\xi^{\prime}=\xi^{\prime}(\xi, \eta), \eta^{\prime}=\eta^{\prime}(\xi, \eta)$ is. conformal by Lemma 4 . Hence $\xi^{\prime} \equiv \xi, \eta^{\prime} \equiv \eta$.

3. A special elliptic system of partial differential equations of the first order. We prove first

TheOREM 1. Let $D(x, y)$ and $E(x, y)$ be of class $L_{2}$ on $\Sigma_{a}=C(0,0 ; a)$ such that 


$$
\iint_{C(P, \rho) \cdot \Sigma_{a}} D^{2} d x d y \leqq M \rho^{\lambda}, \quad \iint_{C(P, \rho) \cdot \Sigma_{a}} E^{2} d x d y \leqq M \rho^{\lambda}, \quad \lambda>0 .
$$

Then the function

$$
U(x, y)=\iint_{\Sigma_{a}} \frac{(\xi-x) D(\xi, \eta)+(\eta-y) E(\xi, \eta)}{(\xi-x)^{2}+(\eta-y)^{2}} d \xi d \eta
$$

is defined and continuous over the whole space, and satisfies a uniform Hölder condition of the form

$$
\begin{array}{r}
\left|U\left(x_{1}, y_{1}\right)-U\left(x_{2}, y_{2}\right)\right| \leqq N r^{\lambda / 2}, N=12(2 \pi M)^{1 / 2}\left[(2-\lambda)^{-1}+\lambda^{-1}(\lambda+1)\right] \\
r=\left[\left(x_{2}-x_{1}\right)^{2}+\left(y_{2}-y_{1}\right)^{2}\right]^{1 / 2}
\end{array}
$$

and

$$
|U(x, y)| \leqq 2\left(1+\lambda^{-1}\right)(2 \pi M)^{1 / 2} \cdot(2 a)^{\lambda / 2}, \quad(x, y) \varepsilon \bar{\Sigma}_{a} .
$$

Proof. Define $D(x, y)=0$ for $x^{2}+y^{2} \geqq a^{2}$ and let

$$
\begin{aligned}
U_{1}(x, y) & =\int_{-\infty}^{\infty} \int_{-\infty}^{\infty} \frac{(\xi-x) \cdot D}{(\xi-x)^{2}+(\eta-y)^{2}} d \xi d \eta \\
& =\iint_{\Sigma_{a}} \frac{(\xi-x) \cdot D}{(\xi-x)^{2}+(\eta-y)^{2}} d \xi d \eta
\end{aligned}
$$

Let

$$
h(x, y ; r)=r^{1 / 2} \int_{0}^{2 \pi}|D(x+r \cos \theta, y+r \sin \theta) \cdot \cos \theta| d \theta
$$

Then

$$
h^{2}(x, y ; r)=r\left[\int_{0}^{2 \pi}|D \cos \theta| d \theta\right]^{2}
$$

By the Hölder inequality $h^{2}(x, y ; r)$ is summable on any interval $0 \leqq r \leqq h$, since

$$
\begin{aligned}
2 \pi \iint_{C(x, y: h)} D^{2} d \xi d \eta=2 \pi \int_{0}^{h} \int_{0}^{2 \pi} r D^{2} d r d \theta & \geqq 2 \pi \int_{0}^{h} r\left[\int_{0}^{2 \pi} D^{2} \cos ^{2} \theta d \theta\right] d r \\
& \geqq \int_{0}^{h} h^{2}(x, y ; r) d r .
\end{aligned}
$$

Thus

$$
\int_{0}^{h} h^{2}(x, y ; r) d r \leqq 2 \pi M h^{\lambda}
$$


If we let $H(x, y ; r)=\int_{0}^{r} h(x, y ; \rho) d \rho$, we find by the Hölder inequality that

$$
H(x, y ; r) \leqq(2 \pi M)^{1 / 2} r^{(1+\lambda) / 2} .
$$

If $N$ is so large that $\Sigma_{a}$ is in the circle $C(x, y ; N)$, then $H(x, y ; r) \leqq\left(2 \pi M N a^{\lambda}\right)^{1 / 2}$ for $r \geqq N$. Hence

$$
\begin{aligned}
\left|\int_{e}^{\rho} \int_{0}^{2 \pi} \frac{(\xi-x) D}{(\xi-x)^{2}+(\eta-y)^{2}} d \xi d \eta\right| \leqq \int_{0}^{\rho} r^{-1 / 2} h(x, y ; r) d r \\
=\rho^{-1 / 2} H(x, y ; \rho)-\epsilon^{-1 / 2} H(x, y ; \epsilon)+\frac{1}{2} \int_{0}^{\rho} r^{-8 / 2} H(x, y ; r) d r \\
\leqq\left(1+\lambda^{-1}\right)(2 \pi M)^{1 / 2} \rho^{\lambda / 2}
\end{aligned}
$$

for every $\epsilon>0$, and every $\rho>0$. Hence $U_{1}(x, y)$ is defined.

Now, let $P_{1}$ and $P_{2}$ be any two points and let $\bar{P}$ be the midpoint of the segment $P_{1} P_{2}$. Let $\rho=\overline{P_{1} P_{2}} / 2$ and let a circle $\sigma(k \rho)$ of radius $k \rho, k>1$, be described about $\bar{P}$ as center. Let $\alpha$ be the inclination of the segment $P_{1} P_{2}$. Then

$$
\begin{aligned}
U_{1}\left(P_{2}\right)-U_{1}\left(P_{1}\right)= & \iint_{\sigma(k \rho)} \frac{\left(\xi-x_{2}\right) D(\xi, \eta)}{\left(\xi-x_{2}\right)^{2}+\left(\eta-y_{2}\right)^{2}} d \xi d \eta \\
& -\iint_{\sigma(k \rho)} \frac{\left(\xi-x_{1}\right) D(\xi, \eta)}{\left(\xi-x_{1}\right)^{2}+\left(\eta-y_{1}\right)^{2}} d \xi d \eta \\
& +\int_{-\rho}^{\rho}\left[\iint_{W-\sigma(k \rho)} \frac{\cos (2 \theta-\alpha) D(\xi, \eta)}{r^{2}} d \xi d \eta\right] d s,
\end{aligned}
$$

where

$$
r^{2}=(\xi-\bar{x}-s \cdot \cos \alpha)^{2}+(\eta-\bar{y}-s \cdot \sin \alpha)^{2}, \quad \theta=\tan ^{-1} \frac{\eta-\bar{y}-s \cdot \sin \alpha}{\xi-\bar{x}-s \cdot \cos \alpha} .
$$

Thus

$$
\begin{aligned}
\left|U_{1}\left(P_{1}\right)-U_{1}\left(P_{2}\right)\right| \leqq & \int_{0}^{(k+1) \rho} r^{-1 / 2} h\left(x_{1}, y_{1} ; r\right) d r+\int_{0}^{(k+1) \rho} r^{-1 / 2} h\left(x_{2}, y_{2} ; r\right) d r \\
& +\int_{-\rho}^{\rho}\left[\int_{(k-1) \rho}^{\infty} r^{-3 / 2} h[x(s), y(s) ; r] d r\right] d s \\
\leqq & 2\left(1+\lambda^{-1}\right)(2 \pi M)^{1 / 2}(k+1)^{\lambda / 2} \rho^{\lambda / 2} \\
& +\int_{-\rho}^{\rho} \frac{3}{2} \int_{(k-1) \rho}^{\infty} r^{-5 / 2} H[x(s), y(s) ; r] d r d s \\
\leqq & 6(2 \pi M)^{1 / 2}\left(1+\lambda^{-1}+[2-\lambda]^{-1}\right) \rho^{\lambda / 2} . \\
& (k=2, x(s)=\bar{x}+s \cos \alpha, y(s)=\bar{y}+s \sin \alpha) .
\end{aligned}
$$


The result follows by using the similar result for $U-U_{1}$.

LeMma 1. Let $D(x, y)$ and $E(x, y)$ be of class $L_{2}$ on $\Sigma_{a}$ with

$$
\iint_{C(P, \rho) \cdot \Sigma_{a}} D^{2} d x d y \leqq M \rho^{\lambda}, \quad \iint_{C(P, \rho) \cdot \Sigma_{a}} E^{2} d x d y \leqq M \rho^{\lambda}, \quad \lambda>0, \quad \rho>0 .
$$

Then there exist sequences $\left\{D_{n}(x, y)\right\}$ and $\left\{E_{n}(x, y)\right\}$ of functions analytic on $\bar{\Sigma}_{a}$ satisfying the above condition with the same $M$ and $\lambda$, and such that

$$
\lim _{n \rightarrow \infty} \iint_{\Sigma_{a}}\left[\left(D_{n}-D\right)^{2}+\left(E_{n}-E\right)^{2}\right] d x d y=0 .
$$

Proof. Define $D(x, y)=E(x, y)=0$ for $x^{2}+y^{2} \geqq a^{2}$, and let $D_{h}$ and $E_{h}$ be the usual average functions. Then $D_{h}$ and $E_{h}$ are continuous on $\Sigma_{a}$ and

$$
\lim _{h \rightarrow \infty} \iint_{\Sigma_{a}}\left[\left(D_{h}-D\right)^{2}+\left(E_{h}-E\right)^{2}\right] d x d y=0,
$$

by Lemma $5, \S 1$. Moreover for each $h>0$

$$
\begin{aligned}
\iint_{C\left(x_{0}, y_{0} ; \rho\right)} D_{h}^{2} d x d y & =\iint_{C\left(x_{0}, y_{0} ; \rho\right)} \frac{1}{16 h^{4}}\left[\int_{x-h}^{x+h} \int_{y-h}^{y+h} D d \xi d \eta\right]^{2} d x d y \\
& \leqq \frac{1}{4 h^{2}} \iint_{C\left(x_{0}, y_{0} ; \rho\right)}\left[\int_{x-h}^{x+h} \int_{y-h}^{y+h} D^{2} d \xi d \eta\right] d x d y \\
& =\frac{1}{4 h^{2}} \int_{-h}^{h} \int_{-h}^{h}\left[\iint_{C\left(x_{0}+\xi, y_{0}+\eta ; \rho\right)} D^{2} d x d y\right] d \xi d \eta
\end{aligned}
$$

the same being true for $E$. The lemma follows easily from this.

Lemma 2. Suppose that $D_{n}, E_{n}, D$, and $E$ are all of class $L_{2}$ on $\Sigma_{a}$, satisfy the condition of Lemma 1, $M$ and $\lambda$ being independent of $n$, and the condition

$$
\lim _{n \rightarrow \infty} \iint_{\Sigma_{a}}\left[\left(D_{n}-D\right)^{2}+\left(E_{n}-E\right)^{2}\right] d x d y=0 .
$$

Then the functions

$$
U_{n}(x, y)=\frac{1}{2 \pi} \iint_{\Sigma_{a}} \frac{(\xi-x) D_{n}+(\eta-y) E_{n}}{(\xi-x)^{2}+(\eta-y)^{2}} d \xi d \eta
$$

converge uniformly on any bounded plane region to the function

$$
U(x, y)=\frac{1}{2 \pi} \iint_{\Sigma_{a}} \frac{(\xi-x) D+(\eta-y) E}{(\xi-x)^{2}+(\eta-y)^{2}} d \xi d \eta
$$


Proof. Since the $U_{n}(x, y)$ are equicontinuous on any bounded set (in fact the whole plane) it is sufficient to prove the convergence at each point $P_{0}:\left(x_{0}, y_{0}\right)$.

Choose $\epsilon>0$, and choose $\rho_{0}$ so small that

$$
\begin{aligned}
& \left|\frac{1}{2 \pi} \iint_{C\left(P_{0}, \rho_{0}\right)} \frac{\left(\xi-x_{0}\right) D_{n}+\left(\eta-y_{0}\right) E_{n}}{\left(\xi-x_{0}\right)^{2}+\left(\eta-y_{0}\right)^{2}} d \xi d \eta\right| \\
& +\left|\frac{1}{2 \pi} \iint_{C\left(P_{0}, \rho_{0}\right)} \frac{\left(\xi-x_{0}\right) D+\left(\eta-y_{0}\right) E}{\left(\xi-x_{0}\right)^{2}+\left(\eta-y_{0}\right)^{2}} d \xi d \eta\right|<\frac{\epsilon}{2} .
\end{aligned}
$$

Then there exists an $N_{0}$ such that for $n>N_{0}$,

$$
\begin{aligned}
\left|\frac{1}{2 \pi} \iint_{\Sigma_{a}-\Sigma_{a} \cdot C\left(P_{0}, \rho_{0}\right)} \frac{\left(\xi-x_{0}\right)\left(D_{n}-D\right)+\left(\eta-y_{0}\right)\left(E_{n}-E\right)}{\left(\xi-x_{0}\right)^{2}+\left(\eta-y_{0}\right)^{2}} d \xi d \eta\right| \\
\quad \leqq \frac{1}{2 \pi \rho_{0}} \iint_{\Sigma_{a}}\left[\left(D_{n}-D\right)^{2}+\left(E_{n}-E\right)^{2}\right]^{1 / 2} d \xi d \eta \\
\leqq\left(2 \rho_{0}\right)^{-1} \cdot \pi^{-1 / 2} \cdot a \cdot\left[\iint_{\Sigma_{a}}\left[\left(D_{n}-D\right)^{2}+\left(E_{n}-E\right)^{2}\right] d \xi d \eta\right]^{1 / 2}<\frac{\epsilon}{2} .
\end{aligned}
$$

This proves the lemma.

Definition 1. Let $D$ and $E$ be functions of class $L_{2}$ on $\Sigma_{a}$. Then if $u$ and $v$ are functions of class $D_{2}$ on $\Sigma_{a}$, we define

$$
\begin{gathered}
K(u, v)=\iint_{\Sigma_{a}} \cdot\left[v_{x}\left(u_{x}+D\right)+v_{y}\left(u_{y}+E\right)\right] d x d y, \\
J(u)=\iint_{\Sigma_{a}}\left[\left(u_{x}+D\right)^{2}+\left(u_{y}+E\right)^{2}\right] d x d y .
\end{gathered}
$$

If the functions $D$ and $E$ have subscripts, we shall denote the integrals formed by using the new functions by putting the same subscripts on $J$ and $K$.

Remarks. It is clear that $J(u+\zeta)=J(u)+2 K(u, \zeta)+D(\zeta)$. Also

$$
\begin{aligned}
& J(u) \leqq 2 D(u)+2 \iint_{\Sigma_{a}}\left(D^{2}+E^{2}\right) d x d y, \\
& D(u) \leqq 2 J(u)+2 \iint_{\Sigma_{a}}\left(D^{2}+E^{2}\right) d x d y .
\end{aligned}
$$

Accordingly, if $H$ is the harmonic function which takes on the same boundary values as $u$, we see that 


$$
\begin{aligned}
& J(u) \leqq J(H) \leqq 2 D(H)+2 \iint_{\Sigma_{a}}\left(D^{2}+E^{2}\right) d x d y ; \\
& D(u) \leqq 4 D(H)+6 \iint_{\Sigma_{a}}\left(D^{2}+E^{2}\right) d x d y .
\end{aligned}
$$

Theorem 2. Let $D$ and $E$ satisfy the conditions of Lemma 1, and let $u^{*}$ be a continuous function defined on $\Sigma_{a}^{*}$ for which $D\left(H^{*}\right)$ is finite, $H^{*}$ being the harmonic function which takes on the given boundary values. Then there exists a unique function $u$ of class $D_{2}$ on $\bar{\Sigma}_{a}$ which takes on these boundary values and minimizes $J(u)$ among all such functions. The function $u(x, y)$ is given by

$$
\begin{aligned}
u(x, y) & =H_{a}(x, y)+U_{a}(x, y), \\
U_{a}(x, y) & =\frac{1}{2 \pi} \iint_{\Sigma_{a}} \frac{(\xi-x) D+(\eta-y) E}{(\xi-x)^{2}+(\eta-y)^{2}} d \xi d \eta,
\end{aligned}
$$

$H_{a}(x, y)$ being the harmonic function which takes on the boundary values $u^{*}-U_{a}$.

Proof. Let $\left\{D_{n}\right\}$ and $\left\{E_{n}\right\}$ be sequences of functions, analytic on $\bar{\Sigma}_{a}$ and satisfying the conditions of Lemma 2 with respect to $D$ and $E$. Let $U(x, y)$ be any function of class $D_{2}$ on $\bar{\Sigma}_{a}$ taking on the given boundary values and let $u_{n}(x, y)$ be the unique solution, for each $n$, of

$$
\Delta u_{n}+D_{n x}+E_{n y}=0 \quad\left(\Delta U=U_{x x}+U_{y y}\right)
$$

which takes on the given boundary values; each $u_{n}$ is the minimizing function for $J_{n}(u)$. Then $J_{n}(U) \rightarrow J(U), J_{n}(U) \geqq J_{n}\left(u_{n}\right)$. Thus $J(U) \geqq \lim \sup _{n \rightarrow \infty} J_{n}\left(u_{n}\right)$.

On the other hand, the functions $u_{n}(x, y)$ are given by

$$
\begin{aligned}
u_{n}(x, y) & =H_{a, n}(x, y)+U_{a, n}(x, y), \\
U_{a, n}(x, y) & =\frac{1}{2 \pi} \iint_{\Sigma_{a}} \frac{(\xi-x) D_{n}+(\eta-y) E_{n}}{(\xi-x)^{2}+(\eta-y)^{2}} d \xi d \eta,
\end{aligned}
$$

where $U_{a, n}(x, y)$ tends uniformly to $U_{a}(x, y)$ so that $H_{a, n}(x, y)$ tends uniformly to $H_{a}(x, y)$ and hence $u_{n}(x, y)$ tends uniformly to the above $u(x, y)$. Since $D\left(u_{n}\right)$ is uniformly bounded, $u(x, y)$ is of class $D_{2}$ and $J(u) \leqq \lim \inf _{n \rightarrow \infty} J_{n}\left(u_{n}\right)$ by Lemma $6, \S 1$. Hence $u(x, y)$ minimizes $J(u)$.

Let $\zeta$ be of class $D_{2}$ on $\bar{\Sigma}_{a}$ and zero on $\Sigma_{a}^{*}$. Then

$$
J(u+\lambda \zeta)=J(u)+2 \lambda K(u, \zeta)+\lambda^{2} D(\zeta) .
$$

Since $u(x, y)$ gives $J$ a minimum, the middle term must vanish. Thus

$$
J(u+\zeta)=J(u)+D(\zeta)>J(u)
$$

unless $\zeta \equiv 0$. 
Remarks. If we let $u_{0, a}(x, y)$ be the minimizing function which is zero on $\Sigma_{a}^{*}$, we see that it is of class $D_{2}$. If $D_{n}$ and $E_{n}$ are as in Lemma 2, it is clear that $u_{0, a, n}$ converges uniformly to $u_{0, a}$ and $D\left(u_{0, a, n}\right)$ is uniformly bounded.

Theorem 3. Let $D$ and $E$ satisfy the conditions of Lemma 1 and let $u$ be the minimizing function for $J(u)$ which takes on given continuous boundary values for which $J(u)$ is finite. Then there exists a unique function $v(x, y)$ which is of class $D_{2}$ on $\Sigma_{a}$, vanishes at the origin, and satisfies

$$
v_{x}=-\left(u_{y}+E\right), \quad v_{y}=u_{x}+D
$$

almost everywhere. Any other function $\bar{V}(x, y)$ which is of class $D_{2}$ and satisfies these relations, differs from $v$ by a constant. The function $v(x, y)$ is given by

$$
\begin{aligned}
v(x, y) & =K_{a}(x, y)+V_{a}(x, y), \\
V_{a}(x, y) & =\frac{1}{2 \pi} \iint_{\Sigma_{a}} \frac{(\xi-x) E-(\eta-y) D}{(\xi-x)^{2}+(\eta-y)^{2}} d \xi d \eta,
\end{aligned}
$$

where $K_{a}(x, y)$ is the conjugate of the $H_{a}(x, y)$ of Theorem 2 .

Proof. Choose $\left\{D_{n}\right\}$ and $\left\{E_{n}\right\}$ as in Lemma.2, let $u_{n}(x, y)$ be the minimizing function for $J_{n}(U)$ with the given boundary values, and let $v_{n}(x, y)$ satisfy

$$
v_{n x}=-\left(u_{n y}+E_{n}\right), \quad v_{n y}=u_{n x}+D_{n} .
$$

Then

$$
\begin{aligned}
v_{n}(x, y) & =K_{a, n}(x, y)+V_{a, n}(x, y), \\
V_{a, n}(x, y) & =\frac{1}{2 \pi} \iint_{\Sigma_{a}} \frac{(\xi-x) E_{n}-(\eta-y) D_{n}}{(\xi-x)^{2}+(\eta-y)^{2}} d \xi d \eta .
\end{aligned}
$$

It is seen by well known methods of differentiating the functions $U_{a, n}$ and $V_{a, n}$ that

$$
V_{a, n, x}=-\left(U_{a, n, y}+E_{n}\right), \quad V_{a, n, y}=U_{a, n, x}+D_{n}
$$

on $\Sigma_{a}$. Thus it is clear that $K_{a, n}(x, y)$ is the conjugate of $H_{a, n}(x, y)$ for each $n$. Since we saw that $H_{a, n}(x, y)$ converged uniformly to $H_{a}(x, y)$ in Theorem 2, and since $V_{a, n}(x, y)$ obviously tends uniformly to $V_{a}(x, y)$, it is clear that $K_{a, n}(x, y)$ tends uniformly to $K_{a}(x, y)$ and $v_{n}(x, y)$ tends uniformly to the above $v(x, y)$ on each closed subregion of $\Sigma_{a}$. Also $D\left(v_{n}\right)=J\left(u_{n}\right)$ and hence $D\left(v_{n}\right)$ is uniformly bounded.

Now, let $\bar{R}$ be a closed subregion of $\Sigma_{a}$. Then $v(x, y)$ is of class $D_{2}$ on $\bar{R}$ with $D(v) \leqq \lim \inf _{n \rightarrow \infty} D\left(v_{n}\right)$ which are uniformly bounded. Hence, by Lemma $6, \S 1$, 


$$
\begin{aligned}
0 & \leqq \iint_{R}\left[\left(v_{x}+u_{y}+E\right)^{2}+\left(v_{y}-u_{x}-D\right)^{2}\right] d x d y \\
& \leqq \liminf _{n \rightarrow \infty} \iint_{R}\left[\left(v_{n x}+u_{n y}+E_{n}\right)^{2}+\left(v_{n y}-u_{n x}-D_{n}\right)^{2}\right] d x d y=0 .
\end{aligned}
$$

Hence $v(x, y)$ satisfies the desired relations almost everywhere. Now if $\bar{V}$ is of class $D_{2}$ and satisfies the same relations almost everywhere, $\bar{V}_{x}-v_{x}=\bar{V}_{y}-v_{y}$ $=0$ almost everywhere so that $\bar{V}-v$ is a constant.

THEOREM 4. If $D$ and $E$ satisfy the conditions of Lemma 1 and $u(x, y)$ and $v(x, y)$ are of class $D_{2}$ on $\Sigma_{a}$ and satisfy

$$
v_{x}=-\left(u_{y}+E\right), \quad v_{y}=u_{x}+D
$$

almost everywhere, then

$$
u(x, y)=H_{a}(x, y)+U_{a}(x, y), \quad v(x, y)=K_{a}(x, y)+V_{a}(x, y),
$$

where $U_{a}$ and $V_{a}$ have their previous significance and $H_{a}$ and $K_{a}$ are conjugate harmonic functions.

Proof. Let $b<a$ so that $v$ is absolutely continuous on $\Sigma_{b}^{*}$, this being true for all values of $b<a$ excepting those in a certain set of measure zero (using Lemma $2, \S 1$ ). Then if $\zeta$ is of class $D_{2}$ on $\bar{\Sigma}_{b}$, we have

$$
\iint_{\Sigma_{b}}\left(\zeta_{x} v_{y}-\zeta_{y} v_{x}\right) d x d y=\int_{\Sigma_{b}^{*}} \zeta d v
$$

Hence if $\zeta$ is also zero on $\Sigma_{b}^{*}$, we see that

$$
\iint_{\Sigma_{b}}\left[\left(u_{x}+D\right) \zeta_{x}+\left(u_{y}+E\right) \zeta_{y}\right] d x d y=\iint_{\Sigma_{b}}\left(\zeta_{x} v_{y}-\zeta_{y} v_{x}\right) d x d y=0 .
$$

Thus $u$ is the minimizing function for $J(u)$ on $\Sigma_{b}$ with $J(u)$ finite, and $v(x, y)$ is its "conjugate" as in Theorem 3. Hence

$$
\begin{aligned}
u(x, y) & =H_{b}^{\prime}(x, y)+\frac{1}{2 \pi} \iint_{\Sigma_{b}} \frac{(\xi-x) D+(\eta-y) E}{(\xi-x)^{2}+(\eta-y)^{2}} d \xi d \eta \\
& =H_{b}(x, y)+U_{a}(x, y), \\
v(x, y) & =K_{b}^{\prime}(x, y)+\frac{1}{2 \pi} \iint_{\Sigma_{b}} \frac{(\xi-x) E-(\eta-y) D}{(\xi-x)^{2}+(\eta-y)^{2}} d \xi d \eta \\
& =K_{b}(x, y)+V_{a}(x, y) .
\end{aligned}
$$

Clearly $H_{b}$ and $K_{b}$ are independent of $b$, and, since $H_{b}^{\prime}$ and $K_{b}^{\prime}$ are conjugate harmonic functions, it is easily seen that $H_{b}$ and $K_{b}$ are. 
4. A more general linear elliptic pair of partial differential equations. In this section, we consider the pair of equations

$$
v_{x}=-\left(b_{2} u_{x}+c u_{y}+e\right), \quad v_{y}=a u_{x}+b_{1} u_{y}+d,
$$

where we assume that $a, b_{1}, b_{2}, c, d$, and $e$ are measurable on a bounded Jordan region $R$ with

(2) $|a|,\left|b_{1}\right|,\left|b_{2}\right|,|c|,|d|,|e| \leqq M, 4 a c-\left(b_{1}+b_{2}\right)^{2} \geqq m>0, a>0$.

Theorem 1. Let $R$ be a bounded Jordan region and $H^{*}$ a function continuous on $\bar{R}$ and harmonic on $R$ for which $D\left(H^{*}\right)$ is finite, and suppose $d=e=0$ on $R$. Then there exists a unique pair of functions $u(x, y)$ and $v(x, y)$

(i) which are of class $D_{2}$ on $\bar{R}$ and $R$ respectively,

(ii) which satisfy (1) almost everywhere on $R$, and

(iii) for which $u(x, y)=H^{*}(x, y)$ on $R^{*}$ and $v\left(x_{0}, y_{0}\right)=0$. These functions also satisfy

(iv) conditions $A\left[2 \lambda, M^{\prime}(\alpha, \delta)\right]$ and $B\left[\lambda, N^{\prime}(\alpha, \delta)\right]$ on $R$ where $\lambda$ depends only on $M$ and $m$, and $M^{\prime}(\alpha, \delta), N^{\prime}(\alpha, \delta)$ depend only on $M, m, \bar{R}$, and the maximum of $\left|H^{*}\right|$ on $\bar{R}$, and the maximum of $|u|$ depends only on the bound for $\left|H^{*}\right|$ on $R^{*}$.

Proof. Approximate to $a, b_{1}, b_{2}, c, d$, and $e$ by sequences $\left\{a_{n}\right\},\left\{b_{1, n}\right\}$, $\left\{b_{2, n}\right\},\left\{c_{n}\right\}$, of functions analytic on $\bar{R}$ for which

$$
\left|a_{n}\right|,\left|b_{1, n}\right|,\left|b_{2, n}\right|,\left|c_{n}\right| \leqq \bar{M}, \quad 4 a_{n} c_{n}-\left(b_{1, n}+b_{2, n}\right)^{2} \geqq \bar{m}>0,
$$

and approximate to $H^{*}$ by harmonic functions $H_{n}^{*}$ for which $D\left(H_{n}^{*}\right) \leqq G$ and such that the solution $u_{n}$ of

$$
\frac{\partial}{\partial x}\left(a_{n} u_{n x}+b_{1, n} u_{n y}\right)+\frac{\partial}{\partial y}\left(b_{2, n} u_{n x}+c_{n} u_{n y}\right)=0
$$

which coincides with $H_{n}^{*}$ on $R^{*}$ is analytic on $\bar{R} \dagger$ for each $n ; \bar{M}, \bar{m}$, and $G$ are independent of $n$. For each $n$, there exists a unique function $v_{n}(x, y)$, with $v_{n}\left(x_{0}, y_{0}\right)=0$, which satisfies

$$
\begin{aligned}
& v_{n x}=-\left(b_{2, n} u_{n x}+c_{n} u_{n y}\right), \\
& v_{n y}=a_{n} u_{n x}+b_{1, n} u_{n y} .
\end{aligned}
$$

Define $A_{n}(x, y), B_{n}(x, y), C_{n}(x, y)$ by

$\dagger$ This can be done by taking a sequence of regions $\bar{R}_{n}$, each bounded by analytic curves, which closes down on $\bar{R}$ and then assigning proper analytic boundary values on $R_{n}^{*}$. That the solutions of (3) exist under these conditions follows from the results stated in the article by $\mathrm{L}$. Lichtenstein on the theory of elliptic partial differential equations in the Encyklopädie der Mathematischen Wissenschaften, vol. II $3^{2}$, pp. 1280-1334. 


$$
\begin{aligned}
& A_{n}=\frac{a_{n}^{2} u_{n x}^{2}+2 a_{n} b_{1, n} u_{n x} u_{n y}+\left(1+b_{1, n}^{2}\right) u_{n y}^{2}}{a_{n} u_{n x}^{2}+\left(b_{1, n}+b_{2, n}\right) u_{n x} u_{n y}+c_{n} u_{n y}^{2}}, \\
& B_{n}=\frac{a_{n} b_{2, n} u_{n x}^{2}+\left(a_{n} c_{n}+b_{1, n} b_{2, n}-1\right) u_{n x} u_{n y}+b_{1, n} c_{n} u_{n y}^{2}}{a_{n} u_{x}^{2}+\left(b_{1, n}+b_{2, n}\right) u_{n x} u_{n y}+c_{n} u_{n y}}, \\
& C_{n}=\frac{\left(1+b_{2, n}^{2}\right) u_{n x}^{2}+2 b_{2, n} c_{n} u_{n x} u_{n y}+c_{n}^{2} u_{n y}^{2}}{a_{n} u_{n x}^{2}+\left(b_{1, n}+b_{2, n}\right) u_{n x} u_{n y}+c_{n} u_{n y}^{2}}
\end{aligned}
$$

where these expressions are defined; otherwise, let $A_{n}=C_{n}=1, B_{n}=0$. It is easily verified that $A_{n}, B_{n}, C_{n}$ are measurable for each $n$ and that

$$
\begin{aligned}
A_{n} C_{n}-B_{n}^{2} & =1,\left|A_{n}\right|,\left|B_{n}\right|,\left|C_{n}\right| \leqq K(\bar{M}, \bar{m}), \\
l \cdot\left(U_{x}^{2}+U_{y}^{2}\right) & \leqq A_{n} U_{x}^{2}+2 B_{n} U_{x} U_{y}+C_{n} U_{n y}^{2} \leqq L \cdot\left(U_{x}^{2}+U_{y}^{2}\right), \\
0<l & \leqq L<\infty,
\end{aligned}
$$

where $K, l$, and $L$ depend only on $\bar{M}$ and $\bar{m}$ (U is any function of class $D_{2}$ on $R$ ).

Let $p, q$, and $r$ be three distinct points on $R^{*}$ and $\pi, \kappa$, and $\rho$ be three distinct points arranged in the same order on $\Sigma^{*}$, the boundary of the unit circle. Map $\bar{R}$ on $\bar{\Sigma}$ by functions $\xi=\xi_{n}(x, y), \eta=\eta_{n}(x, y)$ where $p, q$, and $r$ correspond respectively to $\pi, \kappa$, and $\rho$ and where

$$
\eta_{n x}=-\left(B_{n} \xi_{n x}+C_{n} \xi_{n y}\right), \quad \eta_{n y}=A_{n} \xi_{n x}+B_{n} \xi_{n y} \cdot \dagger
$$

Using the relations (4), (5), and (7) and Theorem 4, §2 we find that $u_{n}(\xi, \eta)$ and $v_{n}(\xi, \eta)$ are of class $D_{2}$ on $\bar{\Sigma}$ and satisfy $v_{n \xi}=-u_{n \eta}, v_{n \eta}=u_{n \xi}$ almost everywhere. They are therefore conjugate harmonic functions (in case $u_{n x}=u_{n y}=0$, it is clear that $v_{n x}=v_{n y}=0$ at almost all of these points; hence at almost all corresponding points, $v_{n \xi}=v_{n \eta}=u_{n \xi}=u_{n \eta}=0$; this takes care of points for which $\left.A_{n}=C_{n}=1, B_{n}=0\right)$. Since the transformations (7) are equicontinuous both ways, a subsequence $\left\{n_{k}\right\}$ of the integers may be chosen so that the corresponding transformations and their inverses converge uniformly to a certain 1-1 continuous transformation of $\bar{R}$ into $\bar{\Sigma}$ and its inverse. Thus the sequences $\left\{u_{n_{k}}(\xi, \eta)\right\}$ and hence $\left\{u_{n_{k}}(x, y)\right\}$ converge uniformly to certain functions $u(\xi, \eta)$ and $u(x, y)$ respectively, $u(x, y)$ coinciding with $H^{*}$ on $R^{*}$ and $u(\xi, \eta)$ being harmonic; and the functions $v_{n_{k}}(\xi, \eta)$ converge uniformly on each closed subregion of $\Sigma$ to $v(\xi, \eta)$, the conjugate of $u(\xi, \eta)$, and so

$\dagger$ It may be shown that $u=u_{n}(x, y), v=v_{n}(x, y)$ carries $\bar{R}$ into a region on a finite sheeted Riemann surface. The transformation (7) merely amounts to mapping this Riemann region conformally on the unit circle $\bar{\Sigma}$ in a $(\xi, \eta)$ plane. We shall use this transformation in the proof of Theorem 2 where this interpretation is not valid. 
$v_{n_{k}}(x, y)$ converge uniformly on each closed subregion of $R$ to a certain function $v(x, y)$.

It is easily verified that (see the proof of Theorem $4, \S 2$ )

$$
\iint_{D}\left(A_{n} U_{x}^{2}+2 B_{n} U_{x} U_{y}+C_{n} U_{y}^{2}\right) d x d y=\iint_{\Delta_{n}}\left(U_{\xi}^{2}+U_{\eta}^{2}\right) d \xi d \eta
$$

for any function $U$ of class $D_{2}$ (in either $(x, y)$ or $(\xi, \eta)$ ), $D$ and $\Delta_{n}$ being corresponding regions of $R$ and $\Sigma$. Since each $u_{n}$ is harmonic, it follows that

$$
\iint_{\Sigma}\left(u_{n \xi}^{2}+u_{n \eta}^{2}\right) d \xi d \eta \leqq \iint_{\Sigma}\left(H_{n \xi}^{* 2}+H_{n \eta}^{* 2}\right) d \xi d \eta \leqq L \cdot G,
$$

where $L$ is the constant in (6) (using relations (6) and (8)). Hence, by (6) and (8), $D\left(u_{n}\right) \leqq L \cdot G / l$ (independent of $n$ ). Thus $u(x, y)$ and $v(x, y)$ are of class $D_{2}$ on $\bar{R}$ and $R$ respectively. Furthermore, by Lemma $6, \S 1$, it follows easily that

$$
\iint_{R}\left[\left(v_{x}+b_{2} u_{x}+c u_{y}\right)^{2}+\left(v_{y}-a u_{x}-b_{1} u_{y}\right)^{2}\right] d x d y=0 .
$$

Thus the existence and properties (i), (ii), and (iii) are established.

To show (iv), define $A, B$, and $C$ by (5) in terms of $a, b_{1}, b_{2}, c$, and $u$, and perform the transformation (7). The relations (6) hold with $M$ and $m$ replacing $\bar{M}$ and $\bar{m}$, and $u(x, y), v(x, y)$ are carried into conjugate harmonic functions. By Theorem $4, \S 2$, the functions $\xi(x, y)$ and $\eta(x, y)$ satisfy conditions $A[2 \lambda, M(\alpha)]$ and $B[\lambda, N(\alpha)]$ where $\lambda$ depends only on $M$ and $m$ and $M(\alpha)$ and $N(\alpha)$ depend only on $\bar{R},(p, q, r),(\pi, \kappa, \rho), M$, and $m$. Thus it is easy to see how to complete the proof of (iv).

Now, suppose $\bar{U}, \bar{V}$ is another pair of functions obeying the conclusions (i), (ii), and (iii). Then $U$ and $V$, where $U=\bar{U}-u, V=\bar{V}-v$, satisfy these conditions with $H^{*} \equiv 0$. By defining $A, B$, and $C$ by (5) in terms of $U, a, b_{1}, b_{2}$, and $c$, and performing the transformation ( 7$)$, we see that $U(\xi, \eta)$ and $V(\xi, \eta)$ are conjugate harmonic functions for which $U(\xi, \eta)=0$ on $\Sigma^{*}$ and $V\left(\xi_{0}, \eta_{0}\right)$ $=0$. Thus $U \equiv V \equiv 0$.

TheOREM 2. Let $\bar{R}$ be a Jordan region and $p, q$, and $r$ be distinct points on $R^{*}$; we assume that $(\bar{R} ; p, q, r)$ satisfies a $D\left(\Lambda, d_{0}\right)$ condition. Let $H^{*}$ be continuous on $\bar{R}$ and harmonic on $R$ with $D\left(H^{*}\right)$ finite. Suppose $a, b_{1}, b_{2}, c, d$, and $e$. satisfy (2) where $d$ and $e$ are not necessarily zero. Then the conclusions of Theorem 1 hold except that (iv) is replaced by

(iv) $u$ and $v$ satisfy a condition $B\left[\lambda, N^{\prime}(\alpha, \delta)\right]$ on $R$ and $|u| \leqq P$ on $R$,

$$
\text { (iv) } \iint_{D}\left(u_{x}^{2}+u_{y}^{2}\right) d x d y \leqq N
$$


for each closed subregion $D$ of $R$; here $\lambda$ depends only on $M, m, \Lambda$, and $d_{0}$, $N^{\prime}(\alpha, \delta)$ and $P$ depend only on these, on $(\bar{R} ; p, q, r ; \pi, \kappa, \rho)$, and on the maximum of $\left|H^{*}\right|$ on $R^{*}$, and $N$ depends only on these and on the distance of $D$ from $R^{*}$.

Proof. Approximate as in Theorem 1 to $a, b_{1}, b_{2}, c, d$, and $e$ by sequences of analytic functions $\left\{a_{n}\right\},\left\{b_{1, n}\right\},\left\{b_{2, n}\right\},\left\{c_{n}\right\},\left\{d_{n}\right\}$, and $\left\{e_{n}\right\}$ and to $H^{*}$ by harmonic functions $H_{n}^{*}$ in such a way that if $u_{n}$ is the solution of

$$
\frac{\partial}{\partial x}\left(a_{n} u_{n x}+b_{1, n} u_{n y}+d_{n}\right)+\frac{\partial}{\partial y}\left(b_{2, n} u_{n x}+c_{n} u_{n y}+e_{n}\right)=0
$$

which coincides on $R^{*}$ with $H_{n}^{*}$, then $u_{n}$ is analytic on $\bar{R}$ and $D\left(H_{n}^{*}\right) \leqq G$, independent of $n$. Define $A_{n}, B_{n}$, and $C_{n}$ by (5) and perform the transformation (7). We find as in Theorem 1 that $u_{n}$ and $v_{n}$ are of class $D_{2}$ in $(\xi, \eta)$ on $\bar{\Sigma}$ and satisfy

$$
v_{n \xi}=-\left(u_{n \eta}+E_{n}\right), \quad v_{n \eta}=u_{n \xi}+D_{n}
$$

almost everywhere, where

$$
D_{n}=d_{n} y_{n \eta}-e_{n} x_{n \eta}, \quad E_{n}=-\left(d_{n} y_{n \xi}-e_{n} x_{n \xi}\right) .
$$

Also

$$
\begin{aligned}
& A_{n} C_{n}-B_{n}^{2}=1,\left|A_{n}\right|,\left|B_{n}\right|,\left|C_{n}\right| \leqq K(\bar{M}, \bar{m}), \\
& l \cdot\left(U_{x}^{2}+U_{y}^{2}\right) \leqq A_{n} U_{x}^{2}+2 B_{n} U_{x} U_{y}+C_{n} U_{y}^{2} \leqq L \cdot\left(U_{x}^{2}+U_{y}^{2}\right), \\
& \iint_{C(P, \rho) \cdot \Sigma}\left(D_{n}^{2}+E_{n}^{2}\right) d \xi d \eta \leqq N_{1}\left(\bar{M}, \bar{m}, \Lambda, d_{0}\right) \cdot \rho^{2 \lambda}, \lambda>0, \lambda=\lambda(\bar{M}, \bar{m}, \Lambda), \\
& \iint_{D}\left(A_{n} U_{x}^{2}+2 B_{n} U_{x} U_{y}+C_{n} U_{y}^{2}\right) d x d y=\iint_{\Delta_{n}}\left(U_{\xi}^{2}+U_{\eta}^{2}\right) d \xi d \eta,
\end{aligned}
$$

where $U$ stands for any function of class $D_{2}$, and $D$ and $\Delta_{n}$ denote corresponding regions of $R$ and $\Sigma$ respectively, and $K, N_{1}$ and $\lambda$ depend only on the quantities indicated, and $l$ and $L$ depend only on $\bar{M}$ and $\bar{m}$. These results are obtained by straightforward computation, the use of Theorems 3 and 4 of $\S 2$, and the relations of Theorem 1 .

Thus

$$
u_{n}=\bar{H}_{n}(\xi, \eta)+u_{0, n}(\xi, \eta),
$$

where $u_{0, n}$ has the significance of $\S 4$ and $\bar{H}_{n}(\xi, \eta)$ is the function harmonic on $\bar{\Sigma}$, and taking on the boundary values of $H_{n}^{*}(\xi, \eta)$. Now, as in Theorem 1, we see that $D\left(u_{n}\right) \leqq L \cdot G / l$ and that a subsequence $\left\{u_{n_{k}}\right\}$ converges uniformly on $\bar{R}$ to a function $u(x, y)$ and $\left\{v_{n_{k}}\right\}$ converges to a function $v(x, y)$ uniformly 
on each closed subregion of $R$. Thus $u$ and $v$ are of class $D_{2}$ and, as in Theorem 1 , are seen to satisfy (1) almost everywhere. It is clear that (i), (ii), and (iii) are fulfilled. That the pair $(u, v)$ is unique follows from Theorem 1 , since if $u^{\prime}$ and $v^{\prime}$ were another pair with the same boundary values, $u^{\prime}-u$ and $v^{\prime}-v$ would satisfy (1) with $d=e=0$ and obey the conclusions (i), (ii), and (iii) of Theorem 1 with $u^{\prime}-u=0$ on $R^{*}$ and $v^{\prime}\left(x_{0}, y_{0}\right)-v\left(x_{0}, y_{0}\right)=0$. That $\left|u_{n}\right| \leqq \bar{P}$ which depends only on $\bar{M}, \bar{m}, \Lambda, d_{0}$, and the maximum of $\left|H^{*}\right|$ on $R^{*}$ follows from Theorems 1 and 2 of $\S 3$.

Using Theorem 3 , $\$ 2$, and (12), we see that (iv) holds except that $\bar{M}$ and $\bar{m}$ intervene instead of $M$ and $m$. Using (11), we see that

$$
\begin{aligned}
\iint_{D}\left(u_{x}^{2}+u_{y}^{2}\right) d x d y & \leqq \liminf _{n \rightarrow \infty} \iint_{D}\left(u_{n \dot{x}}^{2}+u_{n y}^{2}\right) d x d y \\
& \leqq \frac{1}{l} \liminf _{n \rightarrow \infty} \iint_{D}\left(A_{n} u_{n x}^{2}+2 B_{n} u_{n x} u_{n y}+C_{n} u_{n y}^{2}\right) d x d y \\
& =\frac{1}{l} \liminf _{n \rightarrow \infty} \iint_{\Delta_{n}}\left(u_{n \xi}^{2}+u_{n \eta}^{2}\right) d \xi d \eta \leqq N,
\end{aligned}
$$

where $N$ depends on the quantities indicated in the theorem except that $\bar{M}$ and $\bar{m}$ intervene instead of $M$ and $m$; this is true since

$$
\iint_{\Sigma}\left(u_{n, 0 \xi}^{2}+u_{n, 0 \eta}^{2}\right) d \xi d \eta \leqq \iint_{\Sigma}\left(D_{n}^{2}+E_{n}^{2}\right) d \xi d \eta
$$

and regions $D$ at a distance $\geqq \delta$ from $R^{*}$ are carried into regions $\bar{\Delta}_{n}$ at a distance $\geqq m(\delta)$ from $\Sigma^{*}$, where $m(\delta)$ depends only on $\bar{M}, \bar{m}$, and $(\bar{R} ; p, q, r$; $\pi, \kappa, \rho)$ and in such a region $\left|\bar{H}_{n \xi}\right|$ and $\left|\bar{H}_{n \eta}\right| \leqq 2 h_{n} \pi^{-1}[m(\delta)]^{-1}$ where $h_{n}$ denotes the maximum of $\left|H_{n}^{*}\right|$ on $R^{*}$. To get rid of $\bar{M}$ and $\bar{m}$, merely define $A, B, C$ in terms of $a, b_{1}, b_{2}, c$, and $u$ and perform (7); all the conclusions then hold with $M$ and $m$ replacing $\bar{M}$ and $\bar{m}$.

TheOREM 3. Let $\left\{a_{n}\right\},\left\{b_{1, n}\right\},\left\{b_{2, n}\right\},\left\{c_{n}\right\},\left\{d_{n}\right\}$, and $\left\{e_{n}\right\}$ be sequences of measurable functions which converge to $a, b_{1}, b_{2}, c, d$, and e respectively almost everywhere and which satisfy

$$
\begin{aligned}
& \left|a_{n}\right|,\left|b_{1, n}\right|,\left|b_{2, n}\right|,\left|c_{n}\right|,\left|d_{n}\right|,\left|e_{n}\right| \leqq M, \\
& 4 a_{n} c_{n}-\left(b_{1, n}+b_{2, n}\right)^{2} \geqq m>0, \quad a_{n}>0 .
\end{aligned}
$$

For each $n$, let $u_{n}$ and $v_{n}$ be of class $D_{2}$ on $\bar{R}$ and $R$ respectively and satisfy

$$
v_{n x}=-\left(b_{2, n} u_{n x}+c_{n} u_{n y}+e_{n}\right), \quad v_{n y}=a_{n} u_{n x}+b_{1, n} u_{n y}+d_{n}
$$

almost everywhere with $\left|u_{n}\right| \leqq G$ on $R^{*}(M, m$, and $G$ being independent of $n)$. Then 
(i) $|a|,\left|b_{1}\right|,\left|b_{2}\right|,|c|,|d|,|e| \leqq M, 4 a c-\left(b_{1}+b_{2}\right)^{2} \geqq m>0, a>0$,

(ii) $\left\{u_{n}\right\}$ and $\left\{v_{n}\right\}$ are uniformly bounded and satisfy uniform Hölder conditions on each closed subregion $D$ of $R$, which depend only on $M, m, \bar{R}, G$, and the distance $\delta(D)$ of $D$ from $R^{*}$,

(iii) $\iint_{D}\left(u_{n x}+u_{n y}^{2}+v_{n x}^{2}+v_{n y}^{2}\right) d x d y \leqq P[M, m, G, \bar{R}, \delta(D)]$, and

(iv) if the subsequences $\left\{u_{n_{k}}\right\},\left\{v_{n_{k}}\right\}$ are chosen to converge uniformly on each closed subregion $D$ of $R$ to functions $u$ and $v$, then $u$ and $v$ satisfy (ii) and (iii) and

$$
v_{x}=-\left(b_{2} u_{x}+c u_{y}+e\right), \quad v_{y}=a u_{x}+b_{1} u_{y}+d,
$$

almost everywhere on $R$. The same conclusions hold if we merely assume that each $u_{n}$ is of class $D_{2}$ on $R$ with $\left|u_{n}\right| \leqq G$.

Proof. (i) is obvious and (ii) and (iii) have been proved in Theorem 2. To prove (iv), let $D$ be a closed subregion of R. The conclusions (i), (ii), and (iii) hold for $D$ and $\left\{u_{n_{k}}\right\}$ and $\left\{v_{n_{k}}\right\}$ converge uniformly on $\bar{D}$ to $u$ and $v$ which are of class $D_{2}$ on $D$. Then, by Lemma $6, \S 1$,

$$
\begin{aligned}
0 \leqq & \iint_{D}\left[\left(v_{x}+b_{2} u_{x}+c u_{y}+e\right)^{2}+\left(v_{y}-a u_{x}-b_{1} u_{y}-d\right)^{2} d x d y\right. \\
\leqq & \liminf _{n \rightarrow \infty} \iint_{D}\left[\left(v_{n x}+b_{2, n} u_{n x}+c_{n} u_{n y}+e_{n}\right)^{2}\right. \\
& \left.+\left(v_{n y}-a_{n} u_{n x}-b_{1, n} u_{n y}-d_{n}\right)^{2}\right] d x d y=0 .
\end{aligned}
$$

Theorem 4. If $a, b_{1}, b_{2}, c, d$, and e satisfy hypotheses (2) and satisfy $\left|\phi\left(x_{1}, y_{1}\right)-\phi\left(x_{2}, y_{2}\right)\right| \leqq N r^{\lambda}, \quad \lambda>0, \quad r=\left[\left(x_{2}-x_{1}\right)^{2}+\left(y_{2}-y_{1}\right)^{2}\right]^{1 / 2}$, $\left(\phi(x, y)\right.$ standing for $a, b_{1}, b_{2}, c, d$, or $e$ ) on $\bar{R}$, then if $u(x, y)$ and $v(x, y)$ constitute a solution of (1) with $|u| \leqq G$, then $u_{x}, u_{y}, v_{x}$, and $v_{y}$ satisfy a uniform Hölder condition with the same exponent $\lambda$ on any subregion $D$ of $R$ where the constant $N^{\prime}$ depends on $M, m, N, \lambda, \bar{R}$, and the distance of $D$ from $R . \dagger$

5. Applications to the calculus of variations. In this section, we shall discuss the differentiability properties of a function $z(x, y)$ which minimizes

$$
\iint_{R} f(x, y, z, p, q) d x d y \quad\left(p=z_{x}, q=z_{y}\right)
$$

among all functions having the same boundary values. We shall assume that $f$ is continuous together with its first and second partial derivatives for all values of $(x, y, z, p, q)$ and that the second derivatives satisfy a uniform Hölder condition on each bounded portion of $(x, y, z, p, q)$ space, with

$\dagger$ See E. Hopf, loc. cit. 


$$
f_{p p} f_{q q}-f_{p q}^{2}>0, \quad f_{p p}>0
$$

everywhere.

LEMMA $1 . \dagger$ If $z(x, y)$ is a solution of (1) on a region $\bar{R}$, which solution satisfies a uniform Lipschitz condition, then

$$
\int_{D^{*}} f_{p} d y-f_{Q} d x=\iint_{D} f_{z} d x d y,
$$

for almost all rectangles $D$ in $R$.

Proof. Let $\zeta(x, y)$ be any function which satisfies a Lipschitz condition on $\bar{R}$ and which vanishes on $R^{*}$. Then it follows in the usual way that

$$
\iint_{R}\left(\zeta f_{z}+\zeta_{x} f_{p}+\zeta_{y} f_{q}\right) d x d y=0 .
$$

Now let $D: a \leqq x \leqq b, c \leqq y \leqq d$, be any closed rectangle in $R$, and define

$$
\phi(x, y)=\int_{a}^{x} f_{z}[\xi, y, z(\xi, y), p(\xi, y), q(\xi, y)] d \xi .
$$

Then, if $\zeta$ is any function satisfying a uniform Lipschitz condition on $\bar{R}$ and zero outside and on $D^{*}$, we see that

$$
\left.\iint_{D}\left(\zeta \phi_{x}+\zeta_{x} f_{p}+\zeta_{y} f_{q}\right) d x d y=\iint_{D} \zeta_{x}\left(f_{p}-\phi\right)+\zeta_{y} f_{q}\right) d x d y=0
$$

Thus, it follows from a theorem of A. Haar $\ddagger$ that

$$
\int_{\Delta^{*}}\left(f_{p}-\phi\right) d y-f_{q} d x=0
$$

for almost all rectangles $\bar{\Delta}$ of $D$, and hence that

$$
\int_{\Delta^{*}} f_{p} d y-f_{q} d x=\int_{\Delta^{*}} \phi d y=\iint_{\Delta} f_{z} d x d y .
$$

Since $R$ may be written as the sum of a denumerable number of rectangles $D$, the lemma follows.

$\dagger$ This lemma is equivalent to Haar's equations for a minimizing function in a double integral problem, first stated and proved by him in the case that $p$ and $q$ are continuous. See A. Haar, $\ddot{U} b e r$ die Variation der Doppelintegrale, Journal für die Reine und Angewandte Mathematik, vol.149 (1919), pp. 1-18.

‡ A. Haar, Über das Plateausche Problem, Mathematische Annalen, vol. 97 (1926-27), pp. 124158, particularly pp. 146-151. 
THEOREM 1. If $z(x, y)$ satisfies the hypotheses of Lemma 1 , then it is continuous together with its first and second partial derivatives and the latter satisfy uniform Hölder conditions on each closed subregion of $R$. If $f(x, y, z, p, q)$ is analytic, $z(x, y)$ is also.

Proof. Let $h$ be a small rational number and let $\left(x_{0}, y_{0}\right)$ be an interior point of $R$. We define the region $R_{h}$ as the set of all points $\left(x_{1}, y_{1}\right)$ of $R$ such that (1) the segment $x_{1} \leqq x \leqq x_{1}+h, y=y_{1}$ if $h>0$ or $x_{1}+h \leqq x \leqq x_{1}, y=y_{1}$ if $h<0$ lies in $R$ and (2) $\left(x_{1}, y_{1}\right)$ may be joined to $\left(x_{0}, y_{0}\right)$ by a curve, all of whose points satisfy (1). If $|h| \leqq h_{0}, R_{h}$ is a non-vacuous Jordan region ( $h_{0}$ rational). Let $\bar{D}$ be a closed subregion of $R$; for $|h|<h_{1}, R_{h}$ contains $\bar{D}$. Also, if $H$ is any rectangle out of a certain set of "almost all" rectangles of $D$, we have

$$
\begin{aligned}
& \int_{H^{*}}\left\{f_{p}[x+h, y, z(x+h, y), p(x+h, y), q(x+h, y)] d y\right. \\
& \left.\quad-f_{q}[x+h, y, z(x+h, y), p(x+h, y), q(x+h, y)] d x\right\} \\
& =\iint_{H} f_{z}[x+h, y, z(x+h, y), p(x+h, y), q(x+h, y)] d x d y, \\
& \int_{H^{*}}\left\{f_{p}[x, y, z(x, y), p(x, y), q(x, y)] d y\right. \\
& \left.\quad-f_{q}[x, y, z(x, y), p(x, y), q(x, y)] d x\right\} \\
& =\iint_{H} f_{z}[x, y, z(x, y), p(x, y), q(x, y)] d x d y,
\end{aligned}
$$

for all rational $h$ with $|h|<h_{1}$.

Let

$$
z_{h}=\frac{1}{h} \int_{x}^{x+h} z(\xi, y) d \xi .
$$

Then

$$
\begin{aligned}
p_{h}(x, y) & =\frac{z(x+h, y)-z(x, y)}{h}, & q_{h}(x, y) & =\frac{1}{h} \int_{x}^{x+h} q(\xi, y) d \xi, \\
p_{h x} & =\frac{p(x+h, y)-p(x, y)}{h}, & p_{h y} & =\frac{q(x+h, y)-q(x, y)}{h}=q_{h x},
\end{aligned}
$$

almost everywhere $\left(|h|<h_{1}\right)$. Clearly $p_{h}$ satisfies a Lipschitz condition on $D$ for each $h$ with $0<|h|<h_{1}$, and hence is of class $D_{2}$. Also $\left|p_{h}\right|$ is bounded independently of $h$.

Subtracting (3) from (2) and dividing by $h$, we find that 


$$
\begin{aligned}
\int_{H^{*}}\left\{\left[a_{h}(x, y) p_{h x}+b_{h}(x, y) p_{h y}+d_{h}(x, y)\right] d y\right. \\
\left.-\left[b_{h}(x, y) p_{h x}+c_{h}(x, y) p_{h y}+e_{h}(x, y)\right] d x\right\}=0
\end{aligned}
$$

on almost all rectangles $H$ of $D$ (for each rational $h$ with $|h|<h_{1}$ ). Here we may take

$$
\begin{aligned}
a_{h}(x, y)= & \int_{0}^{1} f_{p p}\{x+t h, y, z(x, y)+t[z(x+h, y)-z(x, y)], p(x, y) \\
& +t[p(x+h, y)-p(x, y)], q(x, y)+t[q(x+h, y)-q(x, y)]\} d t, \\
b_{h}(x, y)= & \int_{0}^{1} f_{p q} d t, \quad c_{h}(x, y)=\int_{0}^{1} f_{q q} d t \\
e_{h}(x, y)= & \int_{0}^{1} f_{q x} d t+\frac{z(x+h, y)-z(x, y)}{h} \int_{0}^{1} f_{q z} d t \\
d_{h}(x, y)= & \int_{0}^{1} f_{p x} d t+\frac{z(x+h, y)-z(x, y)}{h} \int_{0}^{1} f_{p z} d t \\
& -\frac{1}{h} \int_{x}^{x+h} f_{z}[\xi, y, z(\xi, y), p(\xi, y), q(\xi, y)] d \xi
\end{aligned}
$$

where the arguments which are not indicated in $b_{h}, c_{h}, d_{h}$, and $e_{h}$ are all the same as that in $a_{h}$. Clearly $\left|a_{h}\right|,\left|b_{h}\right|,\left|c_{h}\right|,\left|d_{h}\right|$, and $\left|e_{h}\right|$ are uniformly bounded for $|h|<h_{1}$ and it can easily be shown as in the proof of Lemma 2 , $\S 2$, that

$$
a_{h} c_{h}-b_{h}^{2} \geqq m>0
$$

for all $h$ with $|h|<h_{1}$. Also from (4), it follows that, for each such $h$, there exists a function $v_{h}(x, y)$ which satisfies a Lipschitz condition on $\bar{R}$ and which satisfies

$$
v_{h}\left(x_{0}, y_{0}\right)=0, \quad v_{h x}=-\left(b_{h} u_{h x}+c_{h} u_{h y}+e_{h}\right), \quad v_{h y}=a_{h} u_{h x}+b_{h} u_{h y}+d_{h}
$$

almost everywhere. Also, if $\left\{h_{n}\right\} \rightarrow 0$ (all rational with $\left.\left|h_{n}\right|<h_{1}\right)\left\{a_{h_{n}}\right\},\left\{b_{h_{n}}\right\}$, $\left\{c_{h_{n}}\right\},\left\{d_{h_{n}}\right\}$, and $\left\{e_{h_{n}}\right\}$ tend to $f_{p p}[x, y, z(x, y), p(x, y), q(x, y)], f_{p q}, f_{q q}$, $f_{p x}+f_{p z} \cdot p-f_{z}$, and $f_{q x}+f_{q z} \cdot p$ respectively. Hence, from Theorem $3, \S 4$ and the fact that $p_{h_{n}}$ tends to $p$ almost everywhere, it follows that $p(x, y)$ satisfies a uniform Hölder condition on each closed subregion $\bar{\Delta}$ of $D$. Similarly it may be shown that $q(x, y)$ also satisfies the same type of condition.

If we choose a subsequence so $\left\{v_{h_{n}}\right\} \rightarrow v$, then we have that

$v_{x}=-\left(f_{p q} p_{x}+f_{q q} q_{x}+\left[f_{q x}+f_{q z} p\right]\right), v_{y}=f_{p p} p_{x}+f_{p q} p_{y}+\left[f_{p x}+f_{p z} p-f_{z}\right]$

almost everywhere. From Theorem $4, \S 4$ it follows that $p_{x}$ and $p_{y}$ satisfy 
uniform Hölder conditions on each subregion of $R$; the same statement holds for $q_{x}$ and $q_{y}$. This proves the theorem. The last statement has been shown to hold by E. Hopf $\dagger$ if it is known that $p(x, y)$ and $q(x, y)$ satisfy Hölder conditions which fact we proved above.

6. Applications to quasi-linear elliptic equations. In this section, we shall consider the equations of the form

$$
a(x, y) z_{x x}+2 b(x, y) z_{x y}+c(x, y) z_{y y}=d(x, y),
$$

(2) $a(x, y, z, p, q) z_{x x}+2 b(x, y, z, p, q) z_{x y}+c(x, y, z, p, q) z_{y y}=\lambda d(x, y, z, p, q)$,

in which we assume that the functions $a, b, c$, and $d$ are defined and continuous for all values of their arguments with $a c-b^{2}=1, a>0$, and that these functions satisfy a uniform Hölder condition in each bounded portion of the space in which they are defined. Then it is known $\ddagger$ that there exists a solution of (1) which is defined in the unit circle $\bar{\Sigma}$ and vanishes on $\Sigma^{*}$ and that its second derivatives satisfy a uniform Hölder condition on each closed subregion of $\Sigma$. A more precise statement is given in Lemma 1 below.

LEMMA 1. Let $z(x, y)$ be the solution of (1) which vanishes on $\Sigma^{*}$, let $k$ be the maximum of $|d| /(a+c)$ on $\bar{\Sigma}$, and let $l$ be the maximum of $(a+c)$ on $\bar{\Sigma}$. Then

$$
|z| \leqq \frac{k}{2}, \quad|p|, \quad|q| \leqq 120 l^{3} k
$$

on $\Sigma$, and $p$ and $q$ satisfy uniform $H$ ölder conditions on each closed subregion $\bar{\Delta}$ of $\Sigma$ which depends only on $k$ and $l$ and the distance of $\bar{\Delta}$ from $\Sigma^{*}$, and $z_{x x}, z_{x y}$, and $z_{y y}$ satisfy uniform $H$ ölder conditions on each closed subregion $\bar{\Delta}$ of $\Sigma$ which depend only on the above and on the Hölder conditions satisfied by $a, b, c$, and $d$.

Proof. First, suppose that $a, b, c$, and $d$ are analytic on $\bar{\Sigma}$ so that $z$ is analytic on $\bar{\Sigma}$. It is known $\S$ that if $d_{1}(x, y) \leqq d(x, y) \leqq d_{2}(x, y)$ on $\bar{R}$ and if $z_{1}$ and $z_{2}$ are the corresponding solutions of (1), then $z_{1} \geqq z \geqq z_{2}$. Hence if we choose

$$
\begin{aligned}
z_{1} & =k\left(1-x^{2}-y^{2}\right) / 2, & z_{2} & =-z_{1}, \\
d_{1}(x, y) & =-k(a+c), & d_{2}(x, y) & =k(a+c),
\end{aligned}
$$

we see that $k / 2 \geqq z \geqq-k / 2$ on $\bar{\Sigma}$. Also, since $z_{1}-z \geqq 0$ and $z-z_{2} \geqq 0$ on $\bar{\Sigma}$, we see that

$\dagger$ Loc. cit.

$\ddagger$ For it is known (see Lichtenstein, loc. cit.) that the solution exists if $a, b, c$, and $d$ are analytic and the result follows from Theorem $4, \S 4$ by approximations.

$\S$ See for instance in S. Bernstein, loc. cit., second paper. 


$$
\frac{\partial z_{2}}{\partial r}=k \geqq \frac{\partial z}{\partial r} \geqq-k=\frac{\partial z_{1}}{\partial r}
$$

on $\Sigma^{*}$. Since $\partial z / \partial \theta=0$ on $\Sigma^{*}$, it follows that

$$
|p|,|q| \leqq k \text { on } \Sigma^{*} \text {. }
$$

Now $p$ and $q$ satisfy the equations

$$
a p_{x}+2 b p_{y}+c q_{y}=d, \quad p_{y}-q_{x}=0 .
$$

If we set $u=-p, v=q$, and $v=p, u=q$, the equations become

$$
\begin{aligned}
& v_{x}=-u_{y}, \quad v_{y}=\frac{a}{c} u_{x}+\frac{2 b}{c} u_{y}+\frac{d}{c}, \\
& v_{x}=-\left(\frac{2 b}{a} u_{x}+\frac{c}{a} u_{y}-\frac{d}{a}\right), \quad v_{y}=u_{x},
\end{aligned}
$$

respectively, each of which is a system of the type treated in $\$ 4$. Thus, by Theorem 3, §4, we see that $p$ and $q$ are bounded and satisfy uniform Hölder conditions as desired in the lemma, and from Theorem $4, \S 4$, we see that $z_{x x}, z_{x y}$, and $z_{y y}$ satisfy the desired conditions; these conditions hold whether $a, b, c$, and $d$ are analytic or not.

To see what the bounds are for $p$ and $q$, let $\bar{R}$ be the unit circle and let $\pi, \kappa, \rho$ and $p, q, r$ be three equally spaced points on $\Sigma^{*}$ and $R^{*}$ respectively. Clearly $(\bar{\Sigma} ; \pi, \kappa, \rho)$ and $(\bar{R} ; p, q, r)$ satisfy $D\left(L, d_{0}\right)$ conditions where $L=4 \pi \cdot 3^{-8 / 2}$ and $d_{0}=3^{1 / 2}$. In making the transformation (7) of $\S 4$, we see that the ratio of the maximum to the minimum magnification at each point is given by $\mu+\left(\mu^{2}-1\right)^{1 / 2}$ where

$$
\begin{aligned}
\mu & =\frac{A+C}{2}=\frac{1}{2} \cdot \frac{\left(1+\frac{a^{2}}{c^{2}}\right) u_{x}^{2}+\frac{4 a b}{c^{2}} u_{x} u_{y}+\left(2+\frac{4 b^{2}}{c^{2}}\right) u_{y}{ }^{2}}{\frac{a}{c} u_{x}^{2}+\frac{2 b}{c} u_{x} u_{y}+u_{y}^{2}} \\
& \leqq \frac{(a+c)^{2}}{2}-1
\end{aligned}
$$

since $a+c \geqq 2$. Thus the $K$ of Theorem $2, \S 2$ is $l^{2}-2$, and hence

$$
\iint_{C\left(P_{0}, r\right) \cdot R}\left(x_{\xi}^{2}+x_{\eta}^{2}+y_{\xi}^{2}+y_{\eta}^{2}\right) d \xi d \eta \leqq 2 \pi\left(l^{2}-2\right)\left(3^{-1 / 2} \cdot 2 r\right)^{2 /\left[\left(l^{2}-2\right)(L+1)\right]} .
$$

Since, on $R, D=(d / c) y_{\eta}, E=-(d / c) y_{\xi}$ in (3) and $D=(d / a) x_{\eta}, E=-(d / a) x_{\xi}$ in (4) (using relations (10) of $\$ 4$ ), it follows that 


$$
\begin{aligned}
& \iint_{C(P, \rho) \cdot R}\left(D^{2}+E^{2}\right) d \xi d \eta \leqq \max _{(x, y) \text { on } \bar{\Sigma}} \frac{4 \pi\left(l^{2}-2\right)}{3^{1 / 2}} \cdot \frac{d^{2}}{c^{2}} \rho^{2 \lambda}, \\
& \iint_{C(P, \rho) \cdot R}\left(D^{2}+E^{2}\right) d \xi d \eta \leqq \max _{(x, y) \in \bar{\Sigma}} \frac{4 \pi\left(l^{2}-2\right)}{3^{1 / 2}} \cdot \frac{d^{2}}{a^{2}} \cdot \rho^{2 \lambda}, \lambda=\frac{1}{\left(l^{2}-2\right)(L+1)^{2}},
\end{aligned}
$$

in cases (3) and (4) respectively. Let us call the first $\gamma \rho^{2 \lambda}$ and the second $\alpha \rho^{2 \lambda}$. Referring to Theorem $1, \S 3$ and using the notations of Theorems 2 and 3 and the remark, we see that

$$
\left|U_{1}\right|,\left|V_{1}\right| \leqq 4\left(1+[2 \lambda]^{-1}\right)\left(\frac{\gamma}{2 \pi}\right)^{1 / 2},\left|U_{1}\right|,\left|V_{1}\right| \leqq 4\left(1+[2 \lambda]^{-1}\right)\left(\frac{\alpha}{2 \pi}\right)^{1 / 2}
$$

in cases (3) and (4) respectively so that

$$
\left|u_{0,1}\right|,\left|v_{0,1}\right| \leqq 8\left(1+[2 \lambda]^{-1}\right)\left(\frac{\gamma}{2 \pi}\right)^{1 / 2},\left|u_{0,1}\right|,\left|v_{0,1}\right| \leqq 8\left(1+[2 \lambda]^{-1}\right)\left(\frac{\alpha}{2 \pi}\right)^{1 / 2}
$$

in (3) and (4) respectively, so that, finally

$$
|u|,|v| \leqq 8\left(1+\frac{1}{2 \lambda}\right)\left(\frac{\gamma}{2 \pi}\right)^{1 / 2}+k,|u|,|v| \leqq 8\left(1+\frac{1}{2 \lambda}\right)\left(\frac{\alpha}{2 \pi}\right)^{1 / 2}+k,
$$

in (3) and (4) respectively. Since $|u|,|v|$ are merely $|p|,|q|$ in one order or the other, and since $2|d| /(a+c)$ is between $|d| / c$ and $|d| / a$, we may substitute $4 k^{2}$ for $d^{2} / a^{2}$ and $d^{2} / c^{2}$ so that we obtain

$$
|p|,|q| \leqq 8\left[1+\frac{1}{2}\left(l^{2}-2\right) \cdot\left(4 \pi \cdot 3^{-3 / 2}+1\right)^{2}\right] 2^{-1 / 2} \cdot 3^{-1 / 4} \cdot l \cdot 2 k \leqq 120 l^{3} k
$$

since $l \geqq 1$.

Definition 1 . A function $z^{*}(x, y)$ is said to satisfy a three point condition with constant $\Delta$ on $\Sigma^{*}$ if for each plane $z=a x+b y+c$ which passes through three points of the curve $z=z^{*}(x, y),(x, y)$ on $\Sigma^{*}$, we have $a^{2}+b^{2} \leqq \Delta^{2}$.

LEMMA 2. Let $z^{*}(x, y)$ satisfy a three point condition with constant $\Delta$ on $\Sigma^{*}$, and let $z$ be the solution of (1) which takes on these boundary values, $d(x, y)$ being assumed to be identically zero on $\bar{\Sigma}$. Then

(i) $\min _{(x, y) \text { on } \Sigma^{*}} z^{*} \leqq z(x, y) \leqq \max _{(x, y) \in \Sigma^{*}} z^{*},(x, y) \varepsilon \bar{\Sigma}$ in $z(x, y)$, and

(ii) $p^{2}+q^{2} \leqq \Delta^{2}$.

Proof. This is well known. $\dagger$

THEOREM 1. Let $z^{*}(x, y)$ satisfy a three point condition with constant $\Delta$ on

$\dagger$ See, for instance, J. Schauder, Über das Dirichletsche Problem im Grossen für nichtlineare elliptische Differentialgleichungen, Mathematische Zeitschrift, vol. 37 (1933), pp. 623-634. 
$\Sigma^{*}$ and let us assume that $\lambda=0$ in equation (2). Then there exists a solution $z(x, y)$ of $(2)$ which coincides with $z^{*}$ on $\Sigma^{*}$.

Proof. Let $M$ denote the maximum of $\left|z^{*}\right|$ on $\Sigma^{*}$ and let $l$ be the least upper bound of $a(x, y, z, p, q)+c(x, y, z, p, q)$ for all functions $z(x, y)$ which satisfy Lipschitz conditions and for which $|z| \leqq M, p^{2}+q^{2} \leqq \Delta^{2}$.

Now, let $z_{0}(x, y)$ be the harmonic function taking on the given boundary values and define functions $z_{n}(x, y)$ for $n \geqq 1$ as the solutions of

$a\left(x, y, z_{n}, p_{n}, q_{n}\right) z_{n+1, x x}+2 b\left(x, y, z_{n}, p_{n}, q_{n}\right) z_{n+1, x y}+c\left(x, y, z_{n}, p_{n}, q_{n}\right) z_{n+1, y y}=0$

which coincide with $z^{*}$ on $\Sigma^{*}$. At each stage

$$
\left|z_{n}\right| \leqq M, \quad p_{n}^{2}+q_{n}^{2} \leqq \Delta^{2}
$$

and $a_{n}, b_{n}, c_{n}$ satisfy Hölder conditions which depend only on $\Delta, M$, and $l$, where $a_{n}$ stands for $a\left(x, y, z_{n}, p_{n}, q_{n}\right)$, for instance. Since $p_{n}$ and $q_{n}$ satisfy the equations

$$
a_{n-1} p_{n x}+2 b_{n-1} p_{n y}+c_{n-1} q_{n y}=0, \quad p_{n y}-q_{n x}=0
$$

and $\left|p_{n}\right|,\left|q_{n}\right| \leqq \Delta$, it follows from $\$ 4$ that $p_{n}$ and $q_{n}$ satisfy uniform Hölder conditions on each closed subregion $D$ of $\Sigma$ which depend only on $l, \Delta$, and the distance of $D$ from $\Sigma^{*}$ (and not on $n$ ). Thus, by Theorem $4, \S 4, z_{n, x x}$, $z_{n, x y}$, and $z_{n, y y}$ satisfy similar conditions, since $a_{n-1}, b_{n-1}$, and $c_{n-1}$ satisfy uniform Hölder conditions on subregions of $\Sigma$ which depend only on the above quantities. Thus a subsequence $\left\{n_{k}\right\}$ may be chosen so that the sequence $\left\{z_{n_{k}}\right\}$ converges uniformly on $\bar{\Sigma}$ to a function $z$ and so that the sequences $\left\{z_{n_{k}, x}\right\}$, $\left\{z_{n_{k}, y}\right\},\left\{z_{n_{k}, x x}\right\},\left\{z_{n_{k}, x y}\right\}$, and $\left\{z_{n_{k}, y y}\right\}$ converge uniformly on each closed subregion of $\Sigma$ to the corresponding derivatives of $z$. Clearly $z$ is the desired solution.

ThEOREM 2. If $|\lambda|$ is sufficiently small, the equation (2) possesses a solution which vanishes on $\Sigma^{*}$.

Proof. Let $l(z)$ and $k(z)$ denote the least upper bounds of

$$
\begin{gathered}
a[x, y, z(x, y), p(x, y), q(x, y)]+c[x, y, z(x, y), p(x, y), q(x, y)], \\
\frac{d[x, y, z(x, y), p(x, y), q(x, y)]}{a[x, y, z(x, y), p(x, y), q(x, y)]+c[x, y, z(x, y), p(x, y), q(x, y)]}
\end{gathered}
$$

respectively. For all $z$ with $z^{2}+p^{2}+q^{2} \leqq \alpha^{2}, l(z) \leqq l, k(z) \leqq k$. Now if $z(x, y)$ is a function for which $z^{2}+p^{2}+q^{2} \leqq \alpha^{2}$ and for which $p(x, y), q(x, y)$ satisfy a uniform Hölder condition on each closed subregion of $\Sigma$, the solution $Z$ of $a(x, y, z, p, q) Z_{x x}+2 b(x, y, z, p, q) Z_{x y}+c(x, y, z, p, q) Z_{y y}=\lambda d(x, y, z, p, q)$ 
which vanishes on $\Sigma^{*}$ is such that $Z_{x x}, Z_{x y}, Z_{y y}$ satisfy Hölder conditions on each closed subregion $D$ of $\Sigma$ which depend only on the Hölder conditions satisfied by $a, b, c, \lambda d$ and $z, p$, and $q$, and $Z_{x}$ and $Z_{y}$ satisfy Hölder conditions which depend only on $\alpha, k, l$, and $\lambda$. Also, by Lemma 1

$$
\left(Z^{2}+P^{2}+Q^{2}\right)^{1 / 2} \leqq 120 \cdot 3^{1 / 2} \cdot l^{3} \cdot k \cdot|\lambda|
$$

which is $\leqq \alpha$ if $\lambda$ is small enough. The successive approximations may be carried through as in Theorem 1.

TheORem 3. Let $L(G)$ and $K(G)$ denote the least upper bounds of $l(z)$ and $k(z)$, respectively, for all $z$ with $z^{2}+p^{2}+q^{2} \leqq G^{2}$. If, in addition to our hypotheses, we assume that

$$
\lim _{G \rightarrow \infty} \frac{L^{8}(G) \cdot K(G)}{G}=0,
$$

the equation (2) possesses a solution on $\Sigma$ which vanishes on $\Sigma^{*}$ for each value of $\lambda$.

Proof. For each $\mu>0$, there exists a number $N_{\mu}$ such that

$$
120 \cdot 3^{1 / 2} \cdot L^{3}(G) \cdot K(G) \leqq \mu G
$$

for all $G \geqq N_{\mu}$. Let $M_{\mu}$ be the least upper bound of $120 L^{3}(G) K(G) \cdot 3^{1 / 2}$ for all $G \leqq N_{\mu}$. If we let $P_{\mu}$ be the larger of $N_{\mu}$ and $\mu^{-1} \cdot M_{\mu}$, we see that

$$
120 l^{3}(z) \cdot k(z) \cdot 3^{1 / 2} \leqq \mu P_{\mu}
$$

for all $z$ for which $z^{2}+p^{2}+q^{2} \leqq P_{\mu}{ }^{2}$. Thus, if $z$ is such a function and $Z$ is the solution of

$a(x, y, z, p, q) Z_{x x}+2 b(x, y, z, p, q) Z_{x y}+c(x, y, z, p, q) Z_{y y}=\lambda d(x, y, z, p, q)$

which vanishes on $\Sigma^{*}$, then

$$
\left(Z^{2}+P^{2}+Q^{2}\right)^{1 / 2} \leqq|\lambda| \cdot \mu \cdot P_{\mu}
$$

which is $\leqq P_{\mu}$ if $|\lambda| \leqq \mu^{-1}$. The remainder of the proof proceeds as in Theorems 1 and 2.

University of California,

Berkeley, Calif. 